\title{
Modulation Instability, Four-Wave Mixing and their Applications
} Tobias Hansson, ${ }^{1}$ Alessandro Tonello, ${ }^{2}$ Stefano Trillo, ${ }^{3}$ and Stefan Wabnitz ${ }^{4}$

\footnotetext{
1 INRS-EMT, 1650 Boulevard Lionel-Boulet, Varennes, Québec, Canada

2 Institut de Recherche XLIM, UMR 7252 CNRS, Université de Limoges, Limoges, France

${ }^{3}$ Department of Engineering, University of Ferrara, Ferrara, Italy

${ }^{4}$ Information Engineering Department, University of Brescia, Brescia, Italy
}

\subsection{Introduction}

Modulation instability (MI) of a continuous wave (CW) background solution of the nonlinear Schrödinger (NLS) equation is a well-known phenomenon that occurs in a variety of fields, such as nonlinear optics, hydrodynamics, plasma physics and Bose-Einstein condensation $[1,2]$. In the nonlinear optics context, $\mathrm{MI}$ is the main mechanism for the generation of optical solitons, supercontinuum (SC) [3,4], and rogue waves [5]. MI may be induced either by quantum noise or by a weak seed signal [6]: in the latter case, the initial stage of exponential signal amplification is followed first by the generation of higher-order sideband pairs by cascade four-photon mixing processes. Next, nonlinear gain saturation occurs, owing to pump depletion. After the maximum level of pump depletion is reached, which depends on the initial sideband detuning, the pump power and the fiber dispersion, energy flows back from the sidebands into the pump, until the initial condition is recovered, and so on. This phenomen provides a classical example of the so-called Fermi-Pasta-Ulam (FPU) recurrence [7,8]. This process can be described in terms of exact solutions of the NLS equation [9-12], and has been experimentally observed in different physical settings, such as deep water waves [13,14], in nonlinear optical fibers [15-20], in nematic liquid crystals [21], magnetic film strip-based active feedback rings [22], and bimodal electrical transmission lines [23]. Important qualitative physical insight into the FPU recurrence dynamics (e.g., the existence of a homoclinic structure and the associated dependence of the FPU recurrence period upon the input relative phase between pump and initial sidebands) may be obtained by means of a truncation to a finite number of Fourier modes, which may lead to simple, low-dimensional models [24-26].

In Section 1.1, we present an overview of the analysis of the nonlinear dynamics of MI by means of a simple three-mode truncation. Next we discuss how the coupling between two polarization modes in a birefringent optical fiber may extend the domain of MI to the normal dispersion regime. Also in the vector case, an important qualitative 
insight into the dynamics of the nonlinear development of MI may be obtained by using a three-mode truncation. We also briefly discuss the case where the MI is induced by two pumps and occurs on top of multiple four-wave mixing. We further present the effect of higher-order MI, which occurs whenever not only the initial modulation seed but also some of its harmonics are modulationally unstable. In this case, at some distance along the fiber one can observe the development of a full modulation of the CW pump with a frequency which is double the initial modulation. A similar situation also occurs in a random birefringence telecom fiber that may be described by means of the Manakov system. In this case, the doubling of the initial modulation frequency occurs whenever the CW pump and the signal are orthogonally polarized. We conclude the first section by examining the competition between spontaneous or noise-induced MI and induced MI, which leads to a break-up of the FPU recurrence after a small number of periods.

MI is a time domain description of a degenerate four-wave mixing (FWM) process in the frequency space. Interestingly, in optical fibers there are a variety of FWM processes: in Section 1.2, we present a peculiar non-degenerate MI process which is also known as Bragg scattering FWM. In this process, two intense pumps lead to a periodic power exchange between a seed signal and an idler, without any exponential amplification of the sidebands. Because of its conservative nature, Bragg scattering FWM has interesting potential applications to quantum optical signal processing.

Finally, in Section 1.3, we present the MI processes that occur in a coherently pumped passive fiber cavity. Again the presence of a phase-sensitive cavity feedback permits the extension of the MI domain into the normal dispersion regime. Moreover, cavity MI is a dissipative type of instability, which leads to the generation of stable trains of optical solitons also known as cavity solitons. We conclude the section by analyzing the MI of the Ikeda map which describes field recirculation in an externally driven cavity in situations (e.g., at high pump powers) that cannot be described by means of the cavityaveraged or mean-field model.

\subsection{Modulation Instability}

In this section, we first present the linear stability analysis of a plane wave background solution of the NLS. Next we generalize the model to include pump depletion and describe a truncated three-wave model for the pump and its immediate sidebands. A phase space description of the nonlinear MI process in terms of Stokes parameters will be introduced and applied to describing the periodic coupling between amplitude and frequency modulated signals. We also discuss the relationship between the temporal MI process, and its equivalent description in the frequency domain in terms of a degenerate four-wave mixing process.

\subsubsection{Linear and Nonlinear Theory of MI}

The NLS equation for the slowly varying envelope $E$ of the electric field that propagates along the distance $z$ in a dispersive fiber is given by

$$
\frac{\partial E(z, \tau)}{\partial z}+i \frac{\beta_{2}}{2} \frac{\partial^{2} E(z, \tau)}{\partial \tau^{2}}-i \gamma|E(t, \tau)|^{2} E(z, \tau)=0
$$

where $\beta_{2}$ is the group velocity dispersion (GVD) coefficient, $\gamma=\omega_{0} n_{2} /\left(c A_{\text {eff }}\right)$ is the nonlinear coefficient, and $\tau$ is a retarded time for a pulse moving at the group velocity at $\omega_{0}$. 
MI of the CW solution of Eq. (1.1) may be analyzed by considering a perturbed solution of the type $E(z, \tau)=\left(\left|E_{0}\right|+u+i v\right) e^{i \operatorname{Arg}\left\{E_{0}\right\}}$. By linearizing Eq. (1.1) around the steadystate $\mathrm{CW}$ solution, one obtains

$$
\left(\tilde{u}_{z}+i \tilde{v}_{z}\right)-i \frac{\beta_{2}}{2} \omega^{2}(\tilde{u}+i \tilde{v})-2 i \gamma\left|E_{0}\right|^{2} \tilde{u}=0
$$

where tilde denotes the Fourier transform with respect to $\tau$. The real and imaginary parts of the previous expression provide two separate linear equations: the possible growth of initial perturbations to the CW background may be investigated by studying the eigenvalues of their coefficient matrix. One finds that the eigenvalues associated with potentially unstable eigenmodes are given by the expression

$$
\lambda= \pm \frac{\left|\beta_{2}\right| \omega}{2} \sqrt{-\operatorname{sgn}\left(\beta_{2}\right) \omega_{c}^{2}-\omega^{2}}
$$

where $\omega_{c}^{2}=4 \gamma\left|E_{0}\right|^{2} /\left|\beta_{2}\right|$. Perturbations will experience growth, and the CW solution experiences $\mathrm{MI}$, at those frequencies where the real part of these eigenvalues is positive. From Eq. (1.3) it is clear that no MI is possible in the normal GVD regime where $\beta_{2}>0$. Whereas for anomalous GVD $\beta_{2}<0$, MI occurs for $\omega<\omega_{c}$, with a maximum exponential growth rate $2 \lambda$ of the sideband powers equal to $2 \gamma\left|E_{0}\right|^{2}$ at the frequency detuning from the pump $\omega_{p}=\omega_{c} / \sqrt{2}$. Physically, this maximum exponential growth is due to the fact that, at this frequency, the four-photon interaction between the pump and the sidebands turns out to be nonlinearly phase matched. Henceforth in this section we consider the anomalous GVD case only.

For the analysis of the nonlinear development of MI past the initial stage of exponential growth of the sidebands, it is convenient to consider a dimensionless version of Eq. (1.1), namely

$$
i \frac{\partial U}{\partial z}+\frac{1}{2} \frac{\partial^{2} U}{\partial t^{2}}+|U|^{2} U=0 .
$$

Here $z$ and $t$ denote dimensionless distance and retarded time, respectively. For the study of the nonlinear development of induced MI, we set the following input condition for Eq. (1.4):

$$
U(z=0, t)=1+\epsilon \exp \left\{i \phi^{0} / 2\right\} \cos (\Omega t)
$$

where $\epsilon \ll 1$, and $\Omega$ is the sideband detuning. In the units of Eq. (1.4), MI occurs for $\Omega<2$, with peak gain for $\Omega=\sqrt{2}$. In real units, the pump power $\left|E_{0}\right|^{2}=\left(\gamma z_{0}\right)^{-1}$, where $z_{0}=\tau_{0}^{2} /\left|\beta_{2}\right|$, and $\tau_{0}=\Omega / \omega$.

Clearly, the linear stability analysis of Eq. (1.2) cannot give any information about the saturation of MI owing to pump depletion. Exact solutions of Eq. (1.4) with the initial condition Eq. (1.5) have been found by direct substitution methods. Quite interestingly, for $\phi^{0}= \pm \pi / 2$, which corresponds to maximum MI gain, the exact solution of the NLS equation is periodic in time $t$ but aperiodic in the distance coordinate $z$. In general, the exact solutions of the NLS equation are either periodic or quasi-periodic in both time $t$ and space $z$. Thus, the aperiodic solution obtained for $\phi^{0}= \pm \pi / 2$ represents a separatrix solution (or homoclinic loop) in the space of time periodic solutions of the NLS equation.

A visualization of the periodic solutions of the NLS equation that are obtained inside, outside and exactly on the separatrix, is provided in Figure 1.1, where the initial frequency modulation $\Omega=\sqrt{2}$. As can be seen in Figure 1.1, the initial weak modulation 


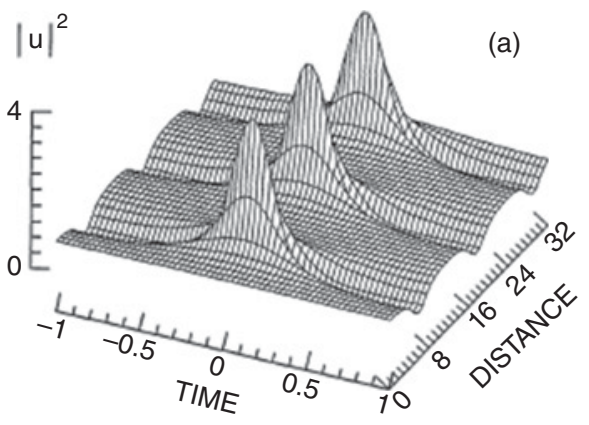

$|u|^{2}$

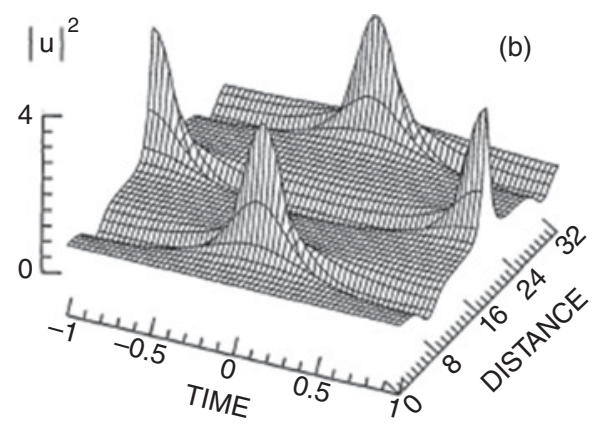

(c)

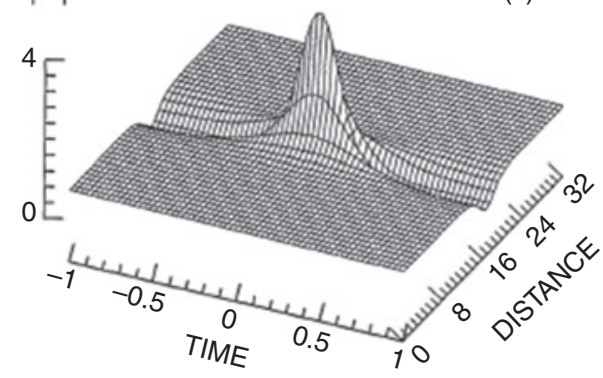

Figure 1.1 Evolutions of a modulated wave. (a) $\epsilon=10^{-2}$ and $\phi^{0}=0$ (amplitude modulation); (b) $\epsilon=10^{-2}$ and $\phi^{0}=\pi$ (frequency modulation); (c) $\epsilon=10^{-4}$ and $\phi^{0}=-\pi / 2$ (separatrix). Source: Trillo 1991 [26].

is amplified until most of the energy is transferred from the pump into the sidebands. Past that point, the flow of energy is reversed and the energy flows back from the sidebands into the pump, until the initial weakly modulated state is recovered, and so on. This periodic behavior of the energy flow among the pump and sidebands provides a typical example of the FPU recurrence effect. Figure 1.1 suggests that the FPU recurrence could be captured by using a finite and possibly small number of Fourier modes $A_{n} \exp (-i n \Omega t)$, with $n=0, \pm 1, \ldots$.

The simplest, yet accurate, model to study the nonlinear evolution of MI is provided by a three-wave truncation $[25,26]$

$$
U(z, t)=A_{0}(z, t)+A_{-1}(z, t) \exp (i \Omega t)+A_{+1}(z, t) \exp (-i \Omega t),
$$

where $A_{0}, A_{-1}$ and $A_{+1}$ are the pump, Stokes and anti-Stokes wave amplitudes, respectively. By inserting Eq. (1.6) in Eq. (1.4), one obtains the three coupled NLS equations

$$
\begin{aligned}
& i \frac{\partial A_{0}}{\partial z}+\frac{1}{2} \frac{\partial^{2} A_{0}}{\partial t^{2}}+\left(\left|A_{0}\right|^{2}+2\left|A_{-1}\right|^{2}+2\left|A_{+1}\right|^{2}\right) A_{0}+2 A_{-1} A_{+1} A_{0}^{*}=0 \\
& i \frac{\partial A_{-1}}{\partial z}-\frac{\Omega^{2}}{2} A_{-1}+i \Omega \frac{\partial A_{-1}}{\partial t}+\frac{1}{2} \frac{\partial^{2} A_{-1}}{\partial t^{2}}+\left(2\left|A_{0}\right|^{2}+\left|A_{-1}\right|^{2}+2\left|A_{+1}\right|^{2}\right) A_{-1}+A_{+1}^{*} A_{0}^{2}=0 \\
& i \frac{\partial A_{+1}}{\partial z}-\frac{\Omega^{2}}{2} A_{+1}-i \Omega \frac{\partial A_{+1}}{\partial t}+\frac{1}{2} \frac{\partial^{2} A_{+1}}{\partial t^{2}}+\left(2\left|A_{0}\right|^{2}+2\left|A_{-1}\right|^{2}+\left|A_{+1}\right|^{2}\right) A_{+1}+A_{-1}^{*} A_{0}^{2}=0
\end{aligned}
$$


The stationary solution $A_{j}(z, t)=\bar{A}_{j}(z)$ of Eqs. (1.7) is exactly integrable by quadratures [25]. We may set $\eta(z)=\left|\bar{A}_{0}(z)\right|^{2} / P_{0}$ and $\phi=\phi_{-1}+\phi_{+1}-2 \phi_{0}$, with $\bar{A}_{j}(z)=$ $\left|\bar{A}_{j}(z)\right| \exp \left\{i \phi_{j}(z)\right\}$, where $P_{0}=\left|\bar{A}_{0}\right|^{2}+\left|\bar{A}_{-1}\right|^{2}+\left|\bar{A}_{+1}\right|^{2}$ is the (conserved) total power of the three waves. Supposing for simplicity that the sidebands have initially equal amplitudes as in Eq. (1.5), i.e., $\bar{A}_{-1}(z=0)=\bar{A}_{+1}(z=0)$, one obtains the following equivalent particle Hamiltonian which describes the spatial evolution of $(\eta, \phi)$

$$
\frac{d \eta}{d Z}=\frac{d H}{d \phi}, \frac{d \phi}{d Z}=-\frac{d H}{d \eta},
$$

where $Z=P_{0} z$, and $H$ is written as

$$
H=2 \eta(1-\eta) \cos (\phi)-(\kappa-1) \eta-\frac{3}{2} \eta^{2}
$$

where $\kappa=-\Omega^{2} / P_{0}$ is a normalized phase mismatch between pump and sidebands. The solutions of Eqs. (1.8) and (1.9) are given in terms of Jacobian elliptic or hyperbolic functions [25]. Even though higher-order sidebands with $|n| \geq 2$ are neglected by the three mode truncation Eq. (1.6) and, accordingly, pump depletion is underestimated, Eqs. (1.8) and (1.9) provide a useful simple model to qualitatively describe the nonlinear dynamics of the induced MI process, as long as the initial sideband detuning is sufficiently large (i.e., $1 \leq \Omega \leq 2$ ), so that the higher-order sidebands are not MI unstable $[24,26]$. The qualitative agreement between the predictions of the three-wave truncated model Eqs. (1.8) and (1.9) and the numerical solutions of the NLS equations (1.4) and (1.5) is illustrated in Figure 1.2. Here we show the solutions of Eq. (1.8) (or curves with constant Hamiltonian $H)$ in the plane $(\eta \sin (\phi), \eta \cos (\phi))$ in the anomalous GVD regime for $\kappa=-2$ or $\Omega=\sqrt{2}$ : note the presence of the separatrix solution which divides two different domains of oscillations in both the analytical and in the numerical solutions.

The validity of the three-mode truncation is based on the fact that, from the numerical solution of Eqs. (1.4) and (1.5), one finds that, at the point of maximum pump depletion which is observed for $\kappa=-1$ or $\Omega=1$, more than $70 \%$ of the pump energy is

(a)

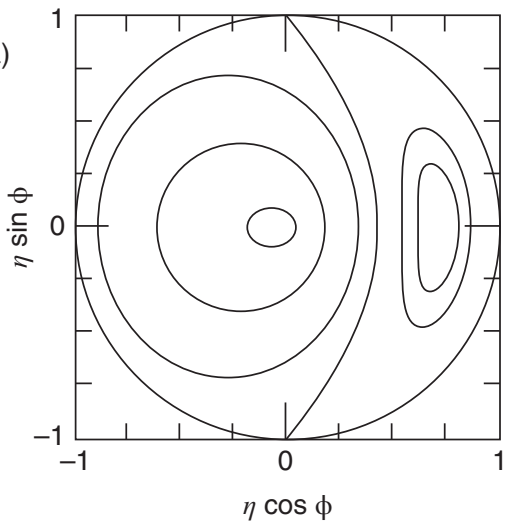

(b)

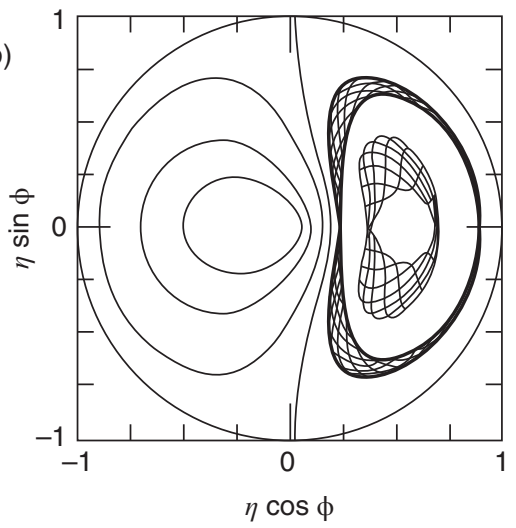

Figure 1.2 Phase space evolutions of nonlinear MI for $\kappa=-2$. (a) truncated three-wave model; (b) numerical solution of the NLS equation. Source: Trillo 1991 [26]. 
converted into the first-order sidebands with $n= \pm 1$ alone. Note that the numerical solutions reveal, in agreement with recent experiments, that maximum pump depletion occurs for a modulation frequency which is lower by a factor of $\sqrt{2}$ than the value corresponding to peak MI gain. Physically this is due to the fact that pump depletion leads to a progressive decrease of the effective optimal MI gain frequency along the fiber.

An alternative geometrical representation of the truncated dynamics of nonlinear modulations is provided by rewriting Eqs. (1.8) and (1.9) in terms of the Stokes parameters $s_{i} \equiv S_{i} / S_{0}$, where $S_{0} \equiv P_{0}=\left|\bar{A}_{0}\right|^{2}+2\left|\bar{A}_{1}\right|^{2}, S_{1} \equiv\left|\bar{A}_{0}\right|^{2}-2\left|\bar{A}_{1}\right|^{2}, S_{2} \equiv \sqrt{2} \bar{A}_{0} \bar{A}_{1}^{*}+$ c.c., and $S_{3} \equiv-i \sqrt{2} \bar{A}_{0} \bar{A}_{1}^{*}+$ c.c. [27]. In vector notation, one obtains

$$
\frac{d \mathbf{s}}{d z}=\left(\Omega_{L}+\Omega_{N L}(\mathbf{s})\right) \times \mathbf{s}
$$

which describes the field evolution on the modulation sphere (analogous to the Poincaré sphere for polarization optics) as the motion of a rigid body subject to the sum of the fixed and the position-dependent angular velocities $\Omega_{L} \equiv(\kappa, 0,0)$ and $\Omega_{N L}=\left(10 s_{1}-\right.$ $\left.1,17 s_{2}, 9 s_{3}\right) / 4$, respectively.

In the modulation sphere shown in Figure 1.3, the two points $s_{1}=( \pm 1,0,0)$ represent the CW and the sidebands, respectively. Whereas the points on the equator with $s_{3}=0$ represent a pure amplitude modulation (AM), and points on the meridian $s_{2}=0$ represent a pure frequency modulation (FM). For $\Omega<2$, MI is present and a separatrix trajectory emanates from the CW (see Figure 1.3 where $\Omega=1$ ). At the same time, two new stable AM eigenmodulations are generated, which are analogous to the elliptically polarized eigenstates that are present in a nonlinear birefringent fiber at high powers. Note that the bifurcation of the sideband mode may also lead to the generation of new stable FM eigenmodulations.

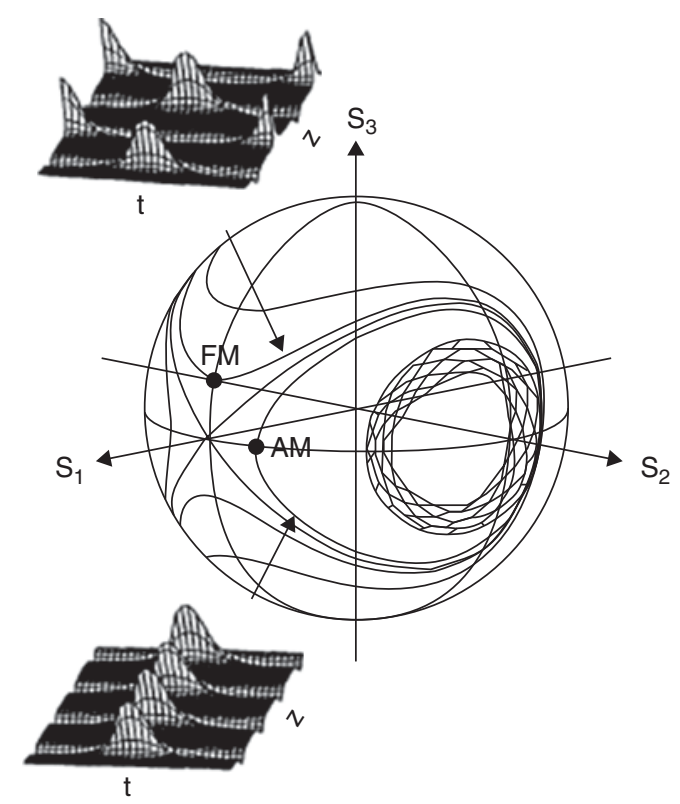

Figure 1.3 AM/FM conversion in MI from the numerical solution of the NLS equation with $\kappa=-1$. Source: Trillo 1991 [27]. 


\subsubsection{Polarization MI (PMI) in Birefringent Fibers}

Based on the coupled NLS equations describing linear and nonlinear coupling in birefringent optical fibers, we present the stability analysis of pump waves with different states of polarization, and show that the range of MI can be extended into the normal dispersion regime. Let us consider for simplicity the case of fibers with low birefringence (with coherent nonlinear coupling terms included). In terms of the circularly polarized components, Eq. (1.1) extends to the system of coherently coupled NLS equations [28]

$$
\begin{aligned}
& \frac{\partial E_{+}}{\partial z}+i \frac{\beta_{2}}{2} \frac{\partial^{2} E_{+}}{\partial \tau^{2}}-\frac{i \Delta \beta}{2} E_{-}-\frac{2 i \gamma}{3}\left[\left|E_{+}\right|^{2}+2\left|E_{-}\right|^{2}\right] E_{+}=0 \\
& \frac{\partial E_{-}}{\partial z}+i \frac{\beta_{2}}{2} \frac{\partial^{2} E_{-}}{\partial \tau^{2}}-\frac{i \Delta \beta}{2} E_{+}-\frac{2 i \gamma}{3}\left[\left|E_{-}\right|^{2}+2\left|E_{+}\right|^{2}\right] E_{-}=0
\end{aligned}
$$

where $\Delta \beta=\beta_{x}-\beta_{y}>0$ is the linear fiber birefringence. In general, the steady-state (i.e, $\tau$-independent) solutions of Eqs. (1.11) can be expressed in terms of $z$-periodic Jacobian elliptic functions. The MI analysis of these solutions can be carried out by computing the spectrum of the associated Floquet exponents over one spatial period. A simpler insight can be obtained by considering the stability analysis of the spatial eigenmodes of Eqs. (1.11), for example, when the state of polarization of the input beam of power $P$ is linear and aligned with the fast axis of the fiber. In terms of the circular polarization components and in the presence of a weak modulation, one has

$$
E_{ \pm}^{f}= \pm\left[i \sqrt{P / 2}+u_{ \pm}+i v_{ \pm}\right] e^{i\left[\gamma P+\beta_{y} z\right]}
$$

By inserting Eqs. (1.12) into Eqs. (1.11) and keeping linear terms only, one obtains

$\frac{\partial \tilde{u}_{+}}{\partial z}+i \frac{\partial \tilde{v}_{+}}{\partial z}-i \frac{\beta_{2}}{2} \omega^{2}\left(\tilde{u}_{+}+i \tilde{v}_{+}\right)-\frac{i \Delta \beta}{2}\left(\tilde{u}_{+}+i \tilde{v}_{+}-\tilde{u}_{-}-i \tilde{v}_{-}\right)-\frac{2}{3} i \gamma P\left(\tilde{v}_{+}+2 \tilde{v}_{+}\right)=0$

along with a similar equation for $\tilde{u}_{-}$and $\tilde{v}_{-}$which can be obtained from Eq. (1.13) by interchanging + with - . By separating the equations for the real and imaginary parts of both circular polarization components of the perturbations, one obtains four separate linear equations. By examining the eigenvalues of the corresponding coefficient matrix, one finds that polarization coupling may lead to MI even in the normal GVD regime, provided that $\omega<\omega_{c 1}$ where $\omega_{c 1}^{2}=\eta(p-1)$ and $p>1$, where $p=P / P_{c}, \eta=2 \Delta \beta /\left|\beta_{2}\right|$ and the critical power $P_{c}=3 \Delta \beta /(2 \gamma)$. The corresponding power growth rate reads as $2 \lambda$, where

$$
\lambda=\frac{\left|\beta_{2}\right|}{2} \sqrt{\left(\eta+\omega^{2}\right)\left(\omega_{c 1}^{2}-\omega^{2}\right)} .
$$

Note that, unlike the scalar MI of Eq. (1.3), in the vector case Eq. (1.14) predicts nonzero gain also for vanishing modulation frequency $\omega=0$, which corresponds to the condition of CW polarization instability of the fast axis. On the other hand, in the anomalous GVD regime, besides experiencing the usual scalar MI as described by Eq. (1.3), the pump wave polarized along the fast axis is MI unstable for $\omega<\omega_{c 2}$ whenever $p>1$, otherwise for $\omega_{c 3}<\omega<\omega_{c 2}$ whenever $p \leq 1$, where $\omega_{c 2}^{2}=\eta$ is the low-power phase matching condition between the pump and the orthogonal sidebands, and $\omega_{c 3}^{2}=\eta(1-p)$. In both the 
normal and anomalous GVD regimes, the described MI is of vector nature, since the unstable Stokes and anti-Stokes eigenmodes are linearly polarized and orthogonal to the pump, that is they are oriented along the slow axis of the fiber [28].

The MI of a pump initially oriented along the slow axis of the fiber can be similarly studied by considering the perturbed eigenmode

$$
E_{ \pm}^{s}=\left[\sqrt{P / 2}+u_{ \pm}+i v_{ \pm}\right] e^{i\left[\gamma P+\beta_{x} z\right]}
$$

By proceeding as outlined before, one finds that in the anomalous GVD regime the slow axis solution Eq. (1.15) is only subject to scalar MI. On the other hand, in the normal GVD regime there is only vector (i.e., with growing sidebands orthogonal to the pump) MI whenever $\omega_{c 2}<\omega<\omega_{c 5}$, with $\omega_{c 5}^{2}=\eta(1+p)$. The corresponding MI gain is obtained from the eigenvalue

$$
\lambda=\frac{\left|\beta_{2}\right|}{2} \sqrt{\left(\omega^{2}-\omega_{c 5}^{2}\right)\left(\omega_{c 2}^{2}-\omega^{2}\right)} .
$$

Therefore with a pump on the slow axis, the MI always occurs at high frequencies above $\omega_{c 2}$, and there is no CW polarization instability.

As we have already done for the scalar case, to study the nonlinear stage of vector MI in a birefringent fiber, we consider the dimensionless version of Eqs. (1.11):

$$
\begin{aligned}
& i \frac{\partial U}{\partial z} \pm \frac{1}{2} \frac{\partial^{2} U}{\partial t^{2}}+\frac{\Delta}{2} U+\left(|U|^{2}+\frac{2}{3}|V|^{2}\right) U+\frac{1}{3} U^{2} V^{*}=0 \\
& i \frac{\partial V}{\partial z} \pm \frac{1}{2} \frac{\partial^{2} V}{\partial t^{2}}-\frac{\Delta}{2} V+\left(|V|^{2}+\frac{2}{3}|U|^{2}\right) V+\frac{1}{3} V^{2} U^{*}=0
\end{aligned}
$$

where $U, V$ are the linear polarization components of the field, and $\Delta$ is the dimensionless linear birefringence [29]. We may then consider the truncation of the solutions of Eqs. (1.17) by using the following ansatz:

$$
\begin{aligned}
& U(z, t)=A_{0}(z) \exp \left(\mp i \Omega^{2} z / 4\right), \\
& V(z, t)=\sqrt{2} A_{1}(z) \exp \left(\mp i \Omega^{2} z / 4\right) \cos (\Omega t) .
\end{aligned}
$$

Accordingly, we now define $\eta(z)=\left|A_{0}(z)\right|^{2} / P_{0}$ and $\phi=2 \phi_{+1}-2 \phi_{0}$, with $A_{j}(z)=$ $\left|A_{j}(z)\right| \exp \left\{i \phi_{j}(z)\right\}, j=0,1$, where $P_{0}=\left|A_{0}\right|^{2}+\left|A_{1}\right|^{2}$ is the total power. The spatial evolution of $(\eta, \phi)$ is again described by the motion of an equivalent particle that obeys the evolution equations [29-31]

$$
\frac{d \eta}{d Z}=\frac{d H}{d \phi}, \frac{d \phi}{d Z}=-\frac{d H}{d \eta}
$$

where $Z=P_{0} z / 3$, and the Hamiltonian $H$ reads as

$$
H=2 \eta(1-\eta) \cos (\phi)-(\kappa+5) \eta+\frac{7}{2} \eta^{2},
$$

where $\kappa=-12\left(\Delta / 2 \pm \Omega^{2} / 4\right) / P_{0}$ is the normalized phase mismatch between pump and sidebands.

We briefly mention that in fibers with high birefringence, a different type of PMI occurs, characterized by growing sidebands which are orthogonally polarized along the 
birefringence axes whereas the pump is linearly polarized at 45 degrees (or more generally at any angle, though the efficiency of the process reduces) [32,33]. This process, which also allows for extending MI to the normal dispersion regime, is described in terms of linearly polarized components $U, V$ by incoherently coupled NLS (IC-NLS) equations where the group-velocity mismatch must be taken into account. The IC-NLS model reads in dimensionless units as

$$
\begin{aligned}
& i \frac{\partial U}{\partial z}+i \delta \frac{\partial U}{\partial t} \pm \frac{1}{2} \frac{\partial^{2} U}{\partial t^{2}}+\left(|U|^{2}+X|V|^{2}\right) U=0 \\
& i \frac{\partial V}{\partial z}-i \delta \frac{\partial U}{\partial t} \pm \frac{1}{2} \frac{\partial^{2} V}{\partial t^{2}}+\left(|V|^{2}+X|U|^{2}\right) V=0
\end{aligned}
$$

where $X=2 / 3$ is the cross-phase modulation (XPM) coefficient in silica fibers. When $\delta$ is sufficiently large, the nonlinear stage of MI can be investigated by means of the two-mode truncation $U=\left[A_{0}(z)+A_{1}(z) \exp (i \Omega t)\right] / \sqrt{2}, V=\left[A_{0}(z)+\right.$ $\left.A_{1}(z) \exp (-i \Omega t)\right] / \sqrt{2}$, where $P_{0}=\left|A_{0}\right|^{2}+\left|A_{1}\right|^{2}$ is the conserved power. Following the same approach outlined above, the dynamics is described by an equivalent integrable oscillator with Hamiltonian [34-36]

$$
H=\frac{2}{3} \eta(1-\eta) \cos (\phi)-(\kappa-1) \eta-\eta^{2}
$$

where $\eta$ and $\phi$ have the same meaning as in Eqs. (1.19) and (1.20). Conversely, in this case, the normalized phase mismatch of the underlying four-photon process reads as $\kappa=\left( \pm \Omega^{2}-\delta \Omega\right) / P_{0}$. When $\delta$ is not large enough, the nature of the MI ruled by Eq. (1.21) changes, since the sidebands possess both polarization components, and six scalar modes become effective. Under this regime the reduced Hamiltonian become two-dimensional and spatially chaotic regimes sets in [35].

\subsubsection{Collective MI of Four-Wave-Mixing}

On the basis of the same type of IC-NLS equations (1.21), it was predicted that MI induced by XPM can occur also by injecting two pumps with the same polarization but different frequencies $\omega_{0} \pm \Omega_{d}$ [37], $2 \Omega_{d}$ being the real-world frequency separation between the pumps. In this case $U$ and $V$ represent the field envelopes at the two carrier frequencies, whereas $X=2$. However, the beating between the two pumps induces, via the Kerr effect, a process usually termed multiple four-wave mixing (mFWM), i.e., the generation of a cascade of multiple sideband pairs at $\omega_{0} \pm n \Omega_{d}, n$ odd integer, which is due to coherent terms neglected in the derivation of Eqs. (1.21) [38]. It was argued that, in the normal GVD regime, the most unstable MI frequencies are resonant with the generated mFWM leading-order modes, which invalidates the approach based on the IC-NLS equations $[39,40]$. However, the dynamics of mFWM could be correctly captured in the framework of the single NLS equation (1.1) and a relative four-mode truncation (pump and the leading-order mFWM mode pairs) [41]. By exploiting the fact that mFWM leads, in a wide range of frequency detuning and powers, to periodic exchange of power between the pump pair and the cascaded modes, the MI linear stability analysis could be reformulated by accounting for the mFWM phenomenon. Indeed, one can linearize around the periodic orbits which describe the mFWM leading-order modes, 

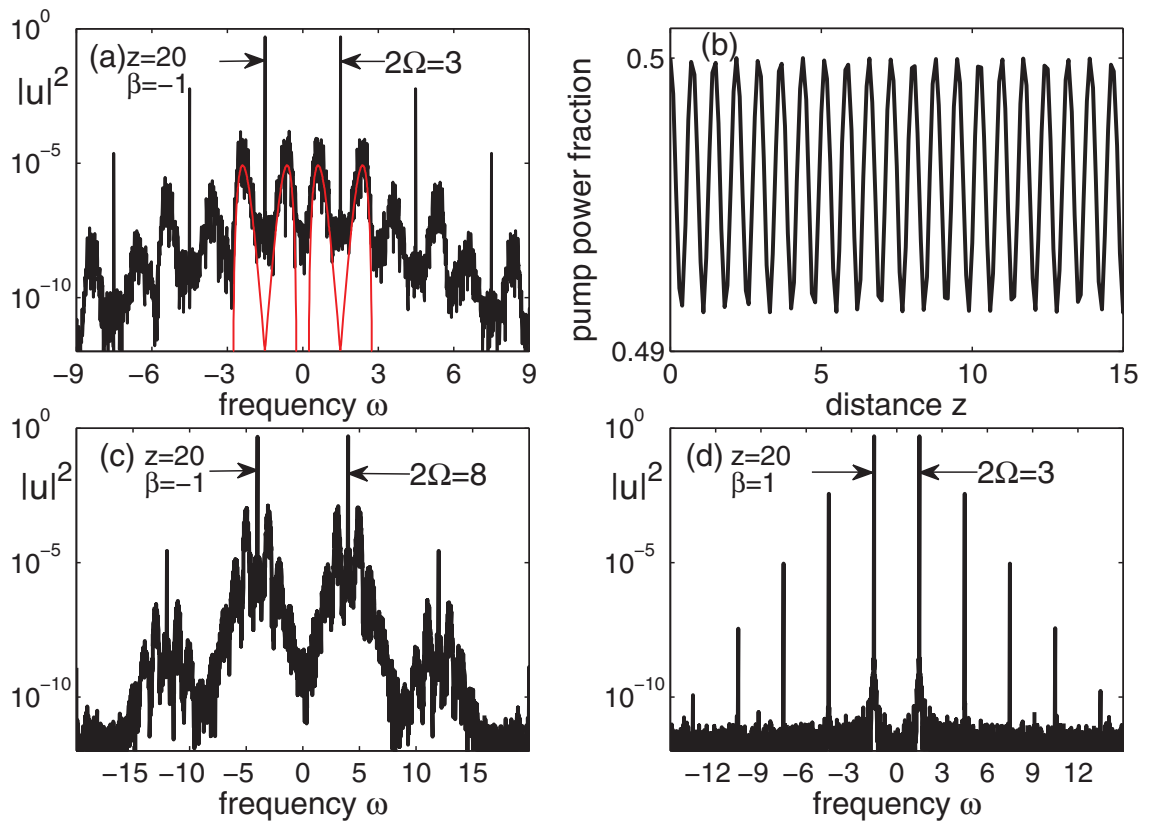

Figure 1.4 (a) Output spectra from a NLS simulation showing the onset of collective MI from noise in the anomalous GVD regime $\left(\beta=\beta_{2} /\left|\beta_{2}\right|=-1\right)$. The frequencies are scaled in units of $\sqrt{\gamma P_{0} / \beta_{2}}$, e.g. $\Omega=\Omega_{d} \sqrt{\beta_{2} /\left(\gamma P_{0}\right)}=1.5$ for the pump detuning. For comparison we report the calculated collective $\mathrm{Ml}$ gain around the pumps (pale gray; the gain peaks at $\omega=\omega_{p}=1$ consistently with the normalized power 0.5 for each pump (normalized total power is 1 ); (b) Corresponding periodic evolution of pump power fraction; (c) as in (a) for larger pump detuning $\Omega=4$; (d) Stable FWM in normal GVD regime, $\beta=1$. Source: Armaroli 2011 [42].

and study by means of Floquet techniques, the stability of the whole process against the growth of a modulation (i.e., sideband pairs) at the same frequency $\delta \omega$ around all the mFWM orders. The outcome of such analysis (for details, see [40,42]) shows that, in the anomalous GVD regime, the $\mathrm{mFWM}$ pattern is unstable against the growth of MI sidebands at the frequency detuning $\delta \omega= \pm \omega_{p}$, where $\omega_{p}=\omega_{c} / \sqrt{2}$ is the peak frequency corresponding to the power of a single pump line, predicted by the standard scalar MI. This process has been termed collective MI because the modulation is transferred with the same frequency from the two pumps to all the other mFWM products. In other words the MI frequency does not scale with the power of the cascaded MFWM modes, as it would be in the event that each mFWM order would develop its own MI [see Figure 1.4 (a)].

An example of the spectrum calculated from the NLS is shown in Figure 1.4 (a). Here the MI develops on top of the spatially periodic evolution of the pumps displayed in Figure 1.4 (b). At larger frequency separations between the pumps also harmonics of the fundamental MI unstable frequency can be observed, as shown in Figure 1.4 (c). The same analysis shows that, for normal GVD, the mFWM is modulationally stable, as shown in Figure 1.4 (d) [40]. These results have been experimentally confirmed, by carefully measuring single spectra as well as the spectral behavior of $\mathrm{mFWM}$, as the frequency detuning $\Omega_{d}$ was continuously varied [43]. 


\subsubsection{Induced MI Dynamics, Rogue Waves, and Optimal Parametric Amplification}

We briefly discuss here the nonlinear dynamics of the evolution of MI beyond the approximation intrinsic in the simple truncated models, that is, including all harmonics $\omega_{0} \pm n \Omega, n \geq 2$ of the initial modulating signal $(n=1)$. These harmonics are indeed generated via the Kerr effect and can be important especially in the scalar MI process. In this case, thanks to the integrability of the NLS equation, the FPU recurrent evolutions that entail a periodic power exchange among the pump and the full comb of harmonics can still be described exactly in terms of doubly-periodic (in time and space) analytical solutions. Such solutions describe the homoclinic structure of the MI illustrated in Figures 1.1 and 1.2. In particular, the separatrix of Eq. (1.4) corresponds to the socalled Akhmediev breather $(\mathrm{AB})$, i.e., a solution that connects the unit background to itself after a full cycle of evolution (strictly speaking, the $A B$ is heteroclinic rather than homoclinic to the background due to the different phase at $z= \pm \infty$ )

$$
u_{A B}(t)=\left[1+\frac{2(1-2 a) \cosh (b z)+i \sinh (b z)}{\sqrt{2 a} \cos (\omega t)-\cosh (b z)}\right] e^{i z}
$$

where the parameters $2 a=\sqrt{1-(\omega / 2)^{2}}$ and $b=\sqrt{8 a(1-2 a)}$ are fixed by the normalized frequency $\omega=\Omega \sqrt{\left|\beta_{2}\right| /\left(\gamma P_{0}\right)}$. Equation (1.23) describes the single cycle of conversion in the whole range of MI, namely $0 \leq \omega \leq 2$ (i.e., $1 / 2 \geq a \geq 0$ ). A remarkable limit of Eq. (1.23) is obtained for $\omega=0$, which gives the rational soliton solution known as the Peregrine soliton [19], a prototype of (deterministic) rogue wave. Moreover for $a>1 / 2$, Eq. (1.23) describes the Kuznetsov-Ma breathers [20]. The nonlinear stage of MI developing from noise usually exhibits evidence for the random excitation of $A B$, Peregrine and Kuznetsov-Ma structures [44]. Conversely, when the MI is induced by a sufficiently small seed, the dynamics can be accurately described in terms of ABs [4, 45], since the doubly periodic solutions lie sufficiently close to the separatrix.

Importantly, from the $\mathrm{AB}$ solutions one can derive a simple analytical condition for the optimum modulation frequency, that leads to maximum pump depletion. This optimum frequency does not coincide with the maximally unstable (or nonlinear phase matching) normalized frequency $\omega=\sqrt{2}$, due to the fact that the depletion tunes the underlying four-photon process out of phase-matching. Conversely, at lower modulation frequencies, even though the modulation grows initially with lower rate, it can be amplified more efficiently since pump depletion tunes the mixing process towards phase matching [25]. Quantitatively, the optimal condition for conversion can be obtained by expanding the $\mathrm{AB}$ at its apex [peak conversion occurring at $z=0$ in Eq. (1.23)] in Fourier series $u_{A B}^{\text {peak }}(t)=\sum_{n} \tilde{u}_{n} \exp (i n \omega t)$, where $\tilde{u}_{n}$ are the Fourier modal amplitudes. In this way, one can obtain simple expressions for the peak fraction of the pump $(n=0)$ and harmonic sideband modes $( \pm n)$

$$
\left|\tilde{u}_{0}^{\text {peak }}\right|^{2}=(\omega-1)^{2} ; \quad\left|\tilde{u}_{n}^{\text {peak }}\right|^{2}=\omega^{2}\left(\frac{2-\omega}{2+\omega}\right)^{n} .
$$

Equation (1.24) implies that the pump is totally depleted at $\omega=1$. As shown in Figure 1.5 (a), numerical integration of the normalized NLS Eq. (1.4) with initial condition $u=\sqrt{\eta_{0}}+\sqrt{1-\eta_{0}} \exp (i \omega t)$, confirms that, at $\omega=1$, total pump depletion occurs in favor of multiple sideband pairs at a characteristic distance $z_{d}$, which depends on 

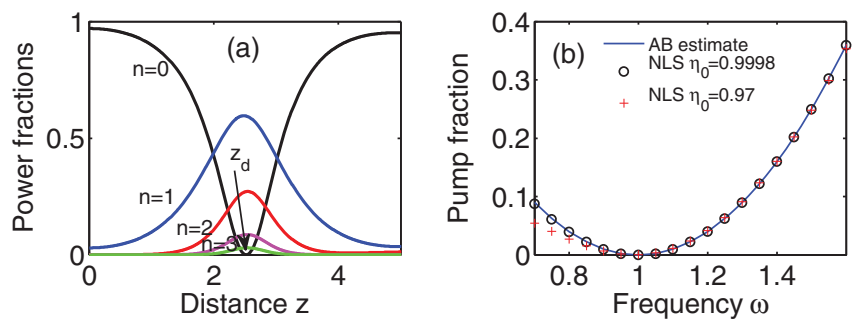

Figure 1.5 (a) Evolution of the power fraction of the pump $(n=0)$ and first four sideband pairs at optimal frequency $\omega=1$ for pump depletion; from numerical integration of NLS equation (1.4); (b) Residual pump power fraction vs. $\omega$ : estimate from AB [Eq. (1.24)], solid line compared with NLS simulations (circles and crosses) for two values of input pump fraction $\eta_{0}=0.97$ and $\eta_{0}=0.9998$.

the power fraction of the input seed. Such simulations, repeated for different modulation frequencies $\omega$, confirm that the parabolic law of Eq. (1.24) indeed provides a quantitatively accurate description of the maximally depleted pump in the whole range $1 \leq \omega \leq 2$, regardless of the initial power fraction of the signal. This agreement is displayed in Figure 1.5 (b), where we compare the results of the simulations, carried out for two different input pump fractions $\eta_{0}=0.9998$ and $\eta_{0}=0.97$ (signal fractions $\eta_{s}=0.02 \%, 3 \%$ ), to the analytical expression [Eq. (1.24)]. As can be seen, slight discrepancies only appear for significantly high input signal fractions (see crosses for $\eta_{0}=0.97$ ), and in the range of modulation frequencies well below the optimum value $\omega=1$. The validity of such arguments has been experimentally validated by measuring a $95 \%$ depletion at a frequency lower than the measured peak gain frequency, in good agreement with the normalized value $\omega=1$ (see Figure 1.6, left panel) [47]. Noteworthy, Figure 1.6 shows that the signal probe $(n=1)$ peaks at a slightly higher frequency (yet lower than
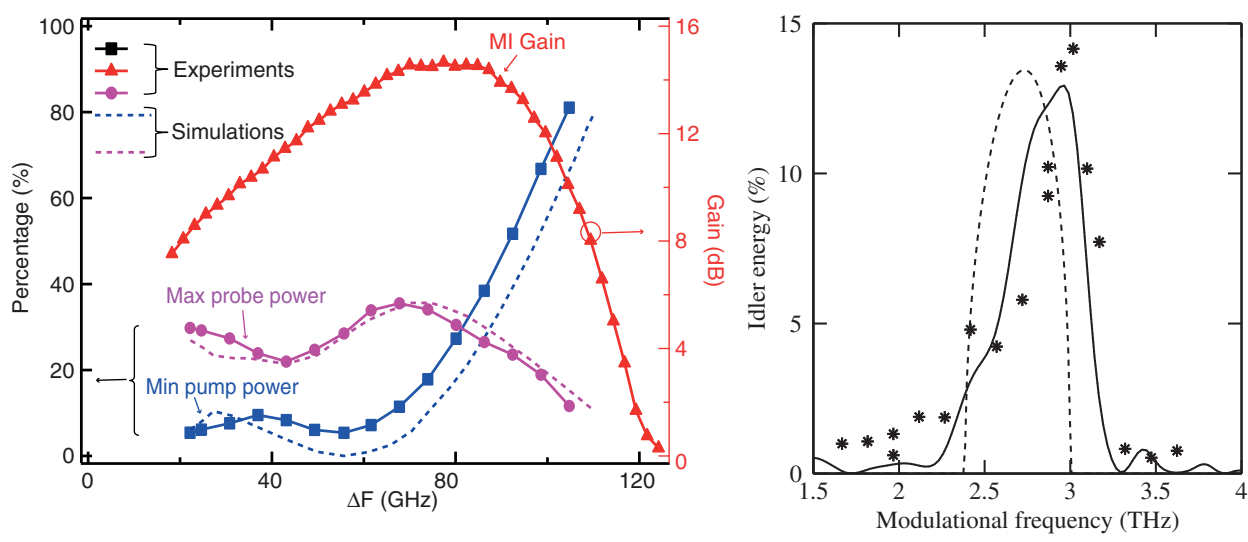

Figure 1.6 Experimental results concerning Ml optimal parametric amplification. Left panel: scalar MI, output pump power and signal fractions compared with small-signal MI gain. Source: Bendahmane 2015 [47]. The dashed lines stand for the corresponding curves from simulations. Right panel: PMI in a highly-birefringent fiber. Output fraction of generated idler vs. modulation frequency, showing a peak conversion at frequency above the cutoff of the PMI gain (dashed), and an abrupt drop above such frequency. The solid line is the numerical result from Eqs. (1.21). The input power fraction of the signal is 0.05. Source: Seve 2000 [36]. Reproduced with permission of American Physical Society. 
the peak gain frequency), in agreement with Eq. (1.24) that gives a maximum of $\left|\tilde{u}_{1}^{\text {peak }}\right|^{2}$ at $\omega \simeq 1.24$.

In the case of PMI processes, the deviation of optimal parametric amplification from nonlinear phase matching is even more striking. Indeed, on the basis of the Hamiltonian oscillators Eqs. (1.20)-(1.22), it was shown that the optimal conversion in the strongly depleted regime occurs outside the gain bandwidth of the MI. Despite the fact that the pump is stable, in this region the dynamics are indeed ruled by unstable phase-locked eigenmodulations, which bifurcate from the pump at the edge of the MI gain bandwidth [29-31]. As a result, a critical frequency turns out to exist, around which the conversion reaches a maximum value and then abruptly drops. In this case the three-mode truncation constitutes a good description of this regime too, since the generation of higherorder sidebands remains negligible. This has been confirmed experimentally in [46] for the low-birefringence case, as well as in [36] for the high-birefringence case. The latter case is illustrated in Figure 1.6 (right panel), which shows the measured idler fraction as a function of modulation frequency compared with the MI gain curve. Here the pump is polarized at 45 degrees and has total power $P=56 \mathrm{~W}$, whereas the input signal is a $10 \%$ fraction of the pump.

\subsubsection{High-Order Induced MI}

Whenever the MI is induced in a fiber by a signal, whose harmonic is also modulationally unstable, then the so-called higher-order MI occurs. In this case, after a first stage of FPU recurrence that leads to the peak pump depletion and maximum amplification of the signal and all of its harmonics, there is a second stage of pump depletion, which is characterized by the development of the second harmonic of the initial modulation [48]. For $0 \leq \Omega \leq 1$, some higher-order sidebands (e.g., the first-harmonic of the input modulation $\pm 2 \Omega$ for $0.5 \leq \Omega \leq 1$ ) experience exponential growth with distance [48]. Consider the solution of Eq. (1.4) with the initial condition Eq. (1.5): Figure 1.7 compares the evolution with distance $\mathrm{z}$ of the field amplitude with either an initial AM (that is $\left.\phi^{0}=0\right)$ or FM $\left(\phi^{0}=\pi\right)$ perturbation. As can be seen, the initial weak modulation grows
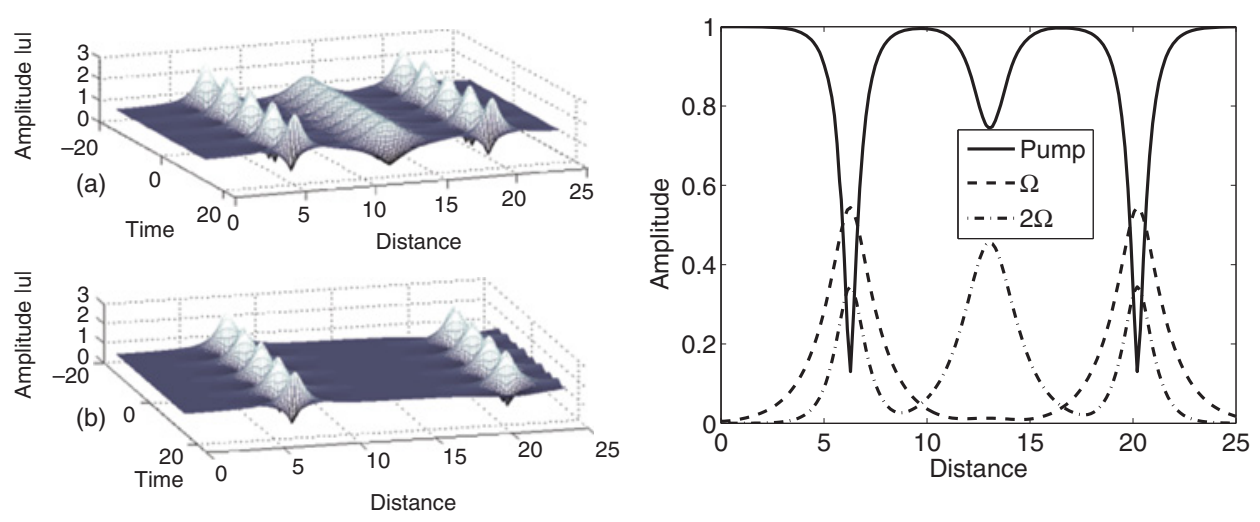

Figure 1.7 Evolution with distance of the field amplitude with $\epsilon=0.01, \Omega=0.8718$, and initial (a) AM or (b) FM. The rightmost plot shows the evolution of the energy in the pump and sidebands for the AM case. Source: Wabnitz 2010 [48]. Reproduced with permission of Elsevier. 
until at $z \simeq 5$ the input CW pump is nearly fully depleted into the sidebands at frequency $\Omega$ and their harmonics. Next, the field energy flows back into the pump according to the usual FPU recurrence.

However, Figure 1.7 (a) also shows that in the AM case, at approximately the midpoint $z \simeq 13$ of the FPU recurrence period, a modulation develops with frequency $2 \Omega$. Whereas Figure 1.7 (b), where a pure FM is present at the fiber input, shows that no development of the second-harmonic component occurs. We may thus conclude that the development of the frequency-doubled modulation is strongly sensitive to the relative phase between the pump and the initial sidebands. Note that the field evolution may be analytically expressed as a nonlinear superposition of all linearly unstable modes, which leads to the emergence of multiple spatial periods $[49,50]$. A potential application of the frequency doubling effect is the possibility of obtaining a high-extinction ratio modulation of a CW laser at frequency $2 \Omega$ by seeding its propagation in a nonlinear optical fiber with a weak modulation at frequency $\Omega$.

An interesting extension of the MI frequency doubling can be obtained by using polarized beams in a randomly birefringent telecom fiber, where pulse propagation is described by the Manakov system

$$
\begin{aligned}
& i \frac{\partial U}{\partial z}+\frac{1}{2} \frac{\partial^{2} U}{\partial t^{2}}+\left(|U|^{2}+|V|^{2}\right) U=0, \\
& i \frac{\partial V}{\partial z}+\frac{1}{2} \frac{\partial^{2} V}{\partial t^{2}}+\left(|V|^{2}+|U|^{2}\right) V=0 .
\end{aligned}
$$

where the initial condition reads as

$$
\begin{aligned}
& U(z=0, t)=U_{0}+\epsilon_{U} \exp \left\{i \phi^{U} / 2\right\} \cos (\Omega t), \\
& V(z=0, t)=V_{0}+\epsilon_{V} \exp \left\{i \phi^{V} / 2\right\} \cos (\Omega t),
\end{aligned}
$$

When the CW pump and its modulation are orthogonally polarized at the fiber input (e.g., with $V_{0}=0$ and $\epsilon_{U}=0$ in Eq. (1.5)), no scalar MI occurs. However, MI is induced upon propagation on the CW via cross-polarization modulation (XPolM) [51]. As a result, as shown in Figure 1.8, one still observes a break-up of the pump into a pulse train which only contains even harmonics of the initial modulation. Moreover, the CW pedestal that accompanies MI-induced pulse trains in the scalar case is fully suppressed in the vector case, thus permitting in principle very large extinction ratios to be achieved. The all-optical generation of a $80-\mathrm{GHz}$ high-contrast pulse train from a cross-polarized 40-GHz electro-optical weak modulation was recently experimentally demonstrated.

\subsubsection{Recurrence Break-Up and Noise}

Although the nonlinear stage of MI is characterized by the FPU recurrence, the development of supercontinuum (SC) is associated with the irreversible evolution toward a thermalization state, i.e., a nearly equal distribution of spectral energy among all frequency components $[3,4]$. For example, it was predicted and experimentally confirmed that third-order dispersion induced losses in Cherenkov radiation lead to the energy dissipation of the pump field, that eventually breaks the FPU recurrence [52,53]. In fact, nonlinear fiber optics experiments typically demonstrate FPU recurrence up to a single spatial period [15-19,47]. In addition, recent studies regarding noise-induced MI have highlighted the complex dynamics associated with the onset stage of noise 

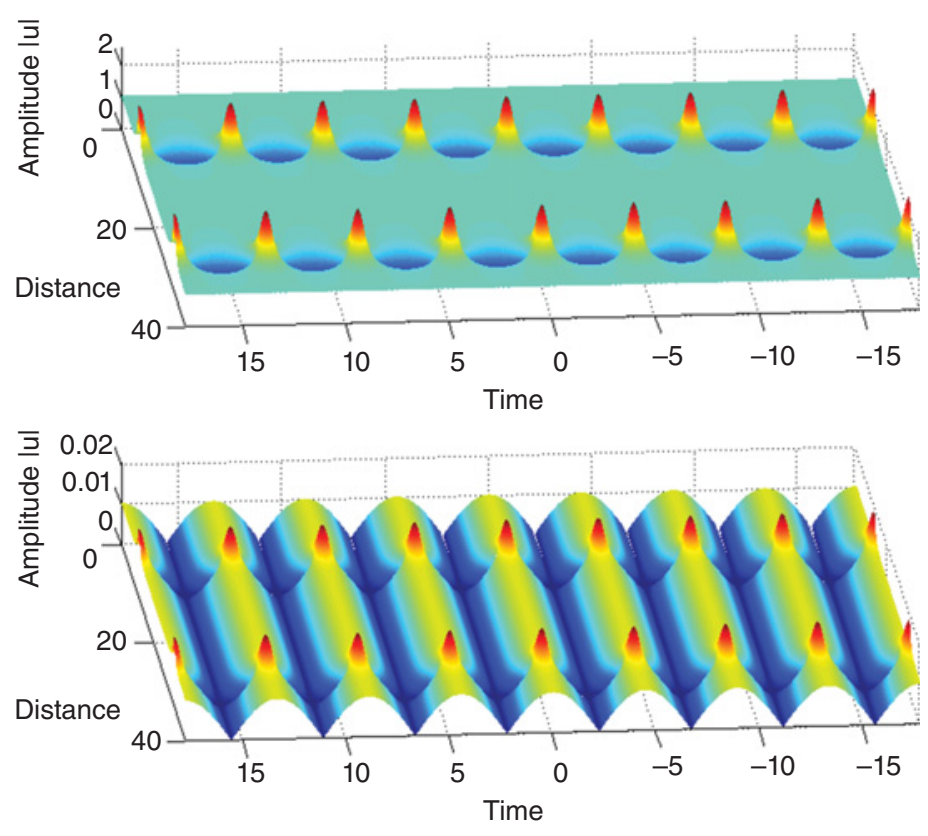

Figure 1.8 Evolution with distance of the field amplitudes $|U|$ and $|V|$ with $U_{0}=1, V_{0}=0, \epsilon_{V}=0.01$, $\phi^{V}=0$ and $\Omega=1 / \sqrt{2}$. Source: Fatome 2013 [51].

amplification $[54,55]$ and their links with the mechanisms of rogue wave generation $[4,56]$. For example, seeding the initial stage of SC generation with a weak modulation could lead to the stabilization of the SC output [56-58], and thus reduce the impact of noise on the complex dynamics associated with $\mathrm{SC}$ and rogue wave generation.

We want to discuss how, in the presence of initial quantum noise, spontaneous $\mathrm{MI}$ competes with the induced MI process. This is a fundamental instability mechanism which breaks the FPU recurrence and leads to the irreversible evolution into statistically stationary spectra [59]. The stability of periodic nonlinear mode coupling was previously studied in the context of polarization MI in birefringent fibers [60], parametric mixing [61] or second harmonic generation in quadratic materials [62], and, more recently, in the closely related problem of dual-frequency pumped $\mathrm{mFWM}$ in optical fibers $[40,42,43]$.

In the following, we numerically solved the NLS equation Eq. (1.4) with the initial condition Eq. (1.5), and added a broadband quantum noise floor corresponding to one photon per frequency bin with random initial phase in the spectral domain. Figure 1.9 (a) shows the evolution of the field intensity $|U(z, t)|^{2}$, for a particular realization of the random input noise seed (single shot case). As can be seen, after just two FPU recurrence periods, spontaneous MI leads to the field break-up into an irregular structure exhibiting frequency doubling and irregular intensity peaks formation.

Break-up of the FPU recurrence is due to the exponential growth of the initial quantum noise background, owing to MI of the periodically evolving pump and multiple FWM sidebands. This is clearly shown in the single-shot spectral domain plot of Figure 1.9 (b), showing the evolution of the log-scale spectral intensity of the field as a function of the angular frequency detuning from the pump, $\omega$. Figure 1.9 (b) also shows 

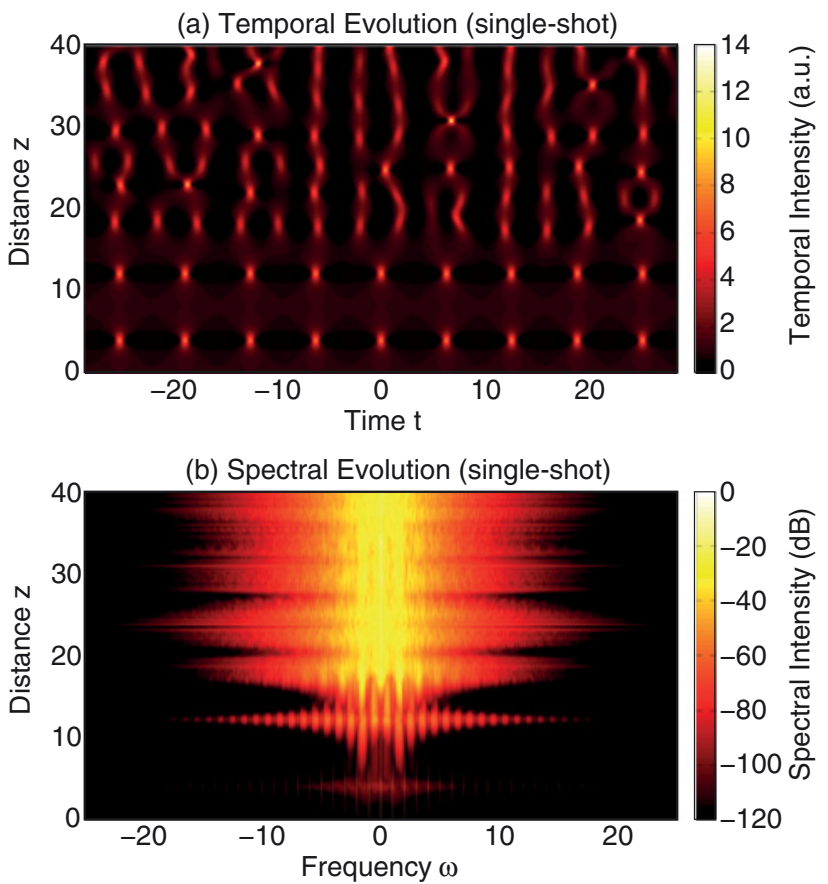

Figure 1.9 Evolution of (a) field intensity $|U|^{2}$; and (b) spectrum (in log scale) for $\Omega=1$ for $\epsilon=0.05$ and $\phi^{0}=0$. Source: Wabnitz 2014 [59].

that, after two periods of the FPU recurrence, the temporal field break-up is associated with the growth of a broad frequency continuum among all FWM sidebands, which leads to the irreversible equipartition of energy in frequency space [59].

The break-up of the FPU recurrence shown in Figure 1.9 is clearly displayed in terms of the spatial evolution of the power of the pump and the initial modulation sidebands. Indeed, Figure 1.10 shows that after two periods of FPU recurrence, the pump power suddenly drops down, and it exhibits an irregular evolution around a low average value.

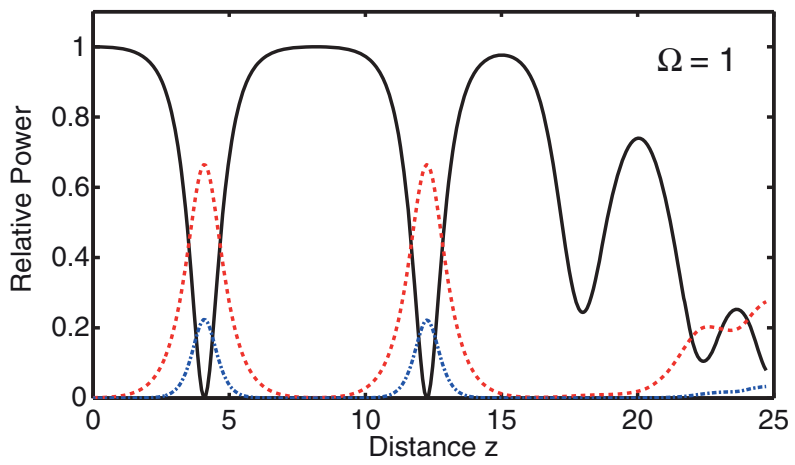

Figure 1.10 Power fraction in the pump (solid black curve) and in the sidebands with frequency shift $\pm \Omega$ (thick dashed curve) or $\pm 2 \Omega$ (thin dashed curve). Source: Wabnitz 2014 [59]. Reproduced with permission of Elsevier. 


\subsection{Four-Wave Mixing Dynamics}

Optical fibers permit the cubic nonlinear response of glass with long interaction lengths to be exploited. If we consider two pump wavelengths at angular frequencies $\omega_{1}$ and $\omega_{2}$, and a third signal at frequency $\omega_{s}$, it is possible, for instance, to generate by FWM an idler at frequency $\omega_{i}=\omega_{1}+\omega_{2}-\omega_{s}$. When the frequencies of the two pumps coincide $\omega_{1}=\omega_{2}$, the FWM is called degenerated. The nearly instantaneous response time of the Kerr nonlinearity makes it very interesting, for instance, for amplification or frequency conversion of optical signals with all-optical devices that are agnostic to the modulation format and directly compatible with fiber transmission systems. However, a real experiment would reveal a more complex situation: for instance, for each pump wavelength, degenerated FWM would also be possible. The efficiency of these processes is governed by different phase matching conditions.

Considering the simple case of a unique guided mode, the nonlinear dynamics of the four interacting waves with two pump wavelengths have been studied in [41] even beyond the common assumption of the undepleted pump approximation, which is instead limited to a weak signal and idler. A variety of four-photon interactions under various polarization states have been predicted and observed in optical fibers [63]; the scenario can be further expanded considering multimode waveguides, where FWM can permit energy exchange among guided modes of different temporal frequencies. The emergence of new technologies for fiber micro structuring has substantially enhanced the possibility of the application of FWM, since the dispersion profile and therefore the phase matching condition and hence the efficiency can be more easily controlled in these fibers.

The FWM process can also naturally be found as an undesired shortcoming in long fiber transmission systems with wavelength division multiplexing: the energy exchange among the interacting waves is in these cases a source of cross-talk among channels and therefore of transmission impairments.

\subsubsection{FWM Processes with Two Pumps}

The first type of application of FWM with two pumps is fiber optic parametric amplification. The same effect can also be obtained with a single pump with the degenerate FWM. It is possible to show that the input signal $\omega_{s}$ can be amplified in an optical fiber, and that at the same time an idler $\omega_{i}$ is created on another wavelength and with the capability of carrying the same information of the signal. The interest in using two pumps is in the gain flatness that one can obtain with the proper choice of pump wavelengths.

Another important application of FWM with two pump wavelengths is the so-called phase-sensitive amplification. The optical amplifiers commonly deployed today in fiber transmission systems are phase-insensitive, which means that they amplify an optical signal, disregarding its input phase. The introduction of more advanced modulation formats, especially phase-shift keying, has increased the interest in new types of optical amplifiers able to amplify the in-phase signal quadrature and to attenuate the out-of-phase signal quadrature. A possible implementation of a phase-sensitive amplification can be based on a FWM comprising a signal wavelength and two pump wavelengths so that $\omega_{s}=\left(\omega_{1}+\omega_{2}\right) / 2$. Some of the features of FWM for phase-sensitive amplification are illustrated in [64], and an example of experimental implementation is given in [65]. 


\subsubsection{Bragg Scattering FWM}

A common feature of the FWM interactions briefly listed in Section 1.3.1 is the presence of an exponential gain. The Bragg-scattering FWM (BS-FWM) is instead a special type of parametric interaction that can permit frequency translations of signals in the absence of exponential gain. The terminology has been inspired by the spatial equivalent effect [66] and this type of FWM was also previously known as wavelength exchange [67].

The absence of gain implies the absence of spontaneous noise emission: for this reason BS-FWM can permit a frequency translation that keeps preserved the quantum state of a signal and this key feature has been extensively developed by McKinstrie and coworkers $[68,69]$.

BS-FWM can in principle permit a unitary transformation involving a weak signal and an idler, with the same formal properties of the transformation operated by a (quantum) beam splitter involving incident reflected and transmitted waves. BS-FWM requires two pumps at two different wavelengths and the maximum conversion efficiency can be obtained at phase matching under various configurations of polarization states and wavelengths for pumps, signal and idler $[68,70,71]$.

In practice, a signal at a given wavelength can be up-converted or down-converted by a frequency-shift amount equal to the beating frequency between two distinct pump wavelengths. The energy conservation requires that $\omega_{i}=\omega_{s} \pm\left(\omega_{1}-\omega_{2}\right)$, where $\omega_{1}, \omega_{2}$ are the angular frequencies of the two pumps $(1,2)$ and $\omega_{s}, \omega_{i}$ are the angular frequencies of signal and idler respectively. The presence of the two signs reminds us that for a given signal wavelength and a pair of pump wavelengths, two distinct idler wavelengths are possible in principle.

Both up-conversion or down-conversion can be implemented in two ways, as illustrated by panels (a) and (b) of Figure 1.11. In the first implementation, shown in panel (a), the pumps are interleaved with the signal and idler, permitting broad frequency translations. In the second implementation, illustrated in panel (b), signal and idler are instead spectrally separated from the two pumps: this configuration permits a narrow-band frequency exchange, and can be of interest in quantum applications where it is important and also technically challenging to separate the pumps from the signal and idler with optical filters.

Considering for simplicity the up-conversion case shown in Figure 1.11 (a), the BS-FWM conversion efficiency is ruled by the phase matching condition

$$
\beta\left(\omega_{i}\right)=\beta\left(\omega_{s}\right)+\left[\beta\left(\omega_{1}\right)-\beta\left(\omega_{2}\right)\right]+\gamma\left(P_{2}-P_{1}\right)
$$

(a)

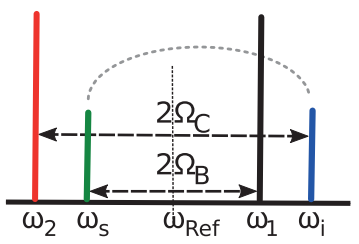

(b)

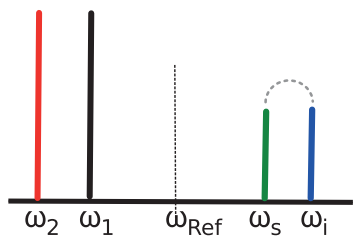

Figure 1.11 Different configurations for BS-FWM. 
where $\beta(\omega)=n(\omega) \omega / c$ is the linear wave-vector at the angular frequency $\omega$ and $P_{1}, P_{2}$ are the powers of pumps 1 and 2. Following [68] it is convenient to identify all interacting waves by frequency detunings $\Omega_{B}, \Omega_{C}$ from a common reference frequency $\omega_{R e f}$. In doing so, it is possible to develop a Taylor series expansion of the wave-vector $\beta(\omega)$ around $\omega_{R e f}$ to highlight the role that is played by the different orders of dispersion. Similar to what happens in the case of MI, the summation of wave-vectors of opposite detuning causes the algebraic cancellation of the odd-order dispersion terms. When the expansion is truncated to the fourth order, the phase matching Eq. (1.27) imposes a condition between the second and the fourth order dispersion as follows:

$$
\left(\Omega_{B}^{2}-\Omega_{C}^{2}\right)\left[\beta_{2}+\frac{\beta_{4}}{12}\left(\Omega_{B}^{2}+\Omega_{C}^{2}\right)\right]=0
$$

In particular, Eq. (1.28) shows that the phase-matching of BS-FWM requires opposite signs for $\beta_{2}$ and $\beta_{4}$ : in normal dispersion $\left(\beta_{2}>0\right)$ it then requires negative values of $\beta_{4}$; in anomalous dispersion instead $\beta_{4}$ should be positive. Both BS-FWM with positive and negative $\beta_{4}$ have been experimentally demonstrated [71].

The dynamical equations coupling the complex envelope of signal $a_{s}$ and idler $a_{i}$ by BS-FWM can be obtained from the standard procedure of analyzing the interplay among two pumps and two sidebands from the NLS equation $[41,66]$. Equations for signal and idler can be linearized in the undepleted pump approximation and the resulting coupled mode equations are

$$
\left[\begin{array}{l}
\frac{d a_{s}}{d z} \\
\frac{d a_{i}}{d z}
\end{array}\right]=\left[\begin{array}{cc}
i \Delta & i \Gamma \\
i \Gamma^{*} & -i \Delta
\end{array}\right]\left[\begin{array}{l}
a_{s} \\
a_{i}
\end{array}\right]
$$

where $2 \Delta=\beta\left(\omega_{s}\right)+\beta\left(\omega_{1}\right)-\beta\left(\omega_{i}\right)-\beta\left(\omega_{2}\right)+\gamma\left(P_{2}-P_{1}\right)$ and $\Gamma=2 \gamma A_{2} A_{1}^{*}$. The corresponding Hamiltonian $H\left(a_{s}, a_{s}^{*}, a_{i}, a_{i}^{*}\right)$ is

$$
H=\Delta\left(\left|a_{s}\right|^{2}-\left|a_{i}\right|^{2}\right)+\Gamma a_{s}^{*} a_{i}+\Gamma^{*} a_{s} a_{i}^{*}
$$

and then the coupled mode Eqs. (1.29) can be obtained directly from $d a_{h} / d z=i \partial H / \partial a_{h}^{*}$, with $h=s, i$.

The solutions of Eqs. (1.29) at a distance $z$ from the origin $z=0$ are

$$
\begin{aligned}
& a_{s}(z)=\mu(z) a_{s}(0)+v(z) a_{i}(0) \\
& a_{i}(z)=-v^{*}(z) a_{s}(0)+\mu^{*}(z) a_{i}(0)
\end{aligned}
$$

with $\mu(z)=\cos (\kappa z)+i \Delta \sin (\kappa z) / \kappa, v(z)=i \Gamma \sin (\kappa z) / \kappa$, and $\kappa^{2}=\Delta^{2}+|\Gamma|^{2}$. At phase matching, the maximum conversion efficiency is obtained when $\kappa z=\pi / 2$ : a slightly longer fiber length would cause a re-translation of the idler toward the signal. BS-FWM has been experimentally demonstrated in highly nonlinear fibers [72] and photonic crystal fibers (PCFs) [69] but has also been reported in other cubic nonlinear materials such as silicon nitride waveguides [73] as well as in Rb-filled PCF [74].

A similar type of noiseless parametric frequency conversion can be obtained in crystals with quadratic nonlinearities by the process of sum-frequency generation: both BS-FWM in fibers and SFG in quadratic crystals can be then represented by an equivalent geometrical interpretation through real-valued Stokes parameters and visualized as a trajectory along the surface of the Poincaré sphere $[75,76]$. 


\subsubsection{Applications of BS-FWM to Quantum Frequency Conversion}

Spontaneous FWM processes have been extensively studied when generating single photons in an all-fiber configuration [77]. Quantum frequency conversions requires instead a combination of a quantum source and a noiseless parametric translation. Quantum frequency translation was first observed by sum-frequency generation in quadratic crystals (see, for instance, [78]).

The first experimental observation of single photon emission and subsequent frequency translation by BS-FWM in an experimental setup entirely based on PCF was reported by McGuinness and coworkers in [69]. To reveal the non-classical nature of the converted signals, besides conversion efficiency and signal to noise ratio, it is necessary to measure correlations. For instance, the authors of [69] measured the conditional second-order degree of coherence $g^{2}(\tau)$, which is proportional to the probability of detecting a second photon at time $t=\tau$ given the fact that a first photon was detected at time $t=0$. The authors measured a value of $g^{2}(0)<1$ that is an indicator of a nonclassical state of light for translated and untranslated light.

Although the process of BS-FWM is intrinsically noiseless, the presence of two pumps generates a series of competitive spontaneous emissions by FWM that can degrade the signal-to-noise ratio in the quantum channels. In optical fibers the spontaneous Raman effect also contributes to generate optical noise at room temperature due to the nature of glass (see, for instance, [70]). Spontanous Raman noise can be reduced by drastically lowering the temperature (for instance, with liquid nitrogen), or by using different materials with lower Raman gain. Silicon has a narrowband Raman gain, and chalcogenide glasses have a spectral window of low spontaneous Raman scattering [79].

\subsection{Fiber Cavity MI and FWM}

\subsubsection{Dynamics of MI in a Passive Fiber Cavity}

A fiber ring cavity is a simple optical device that can be made by connecting the two ends of an optical fiber into a loop configuration using a beam splitter or a fiber coupler. The fiber ring allows light to recirculate inside the cavity over multiple round trips to create an optical resonator. In the following we will focus on passive fiber cavities that are pumped using an external continuous wave $(\mathrm{CW})$ laser source and that do not contain any gain medium. This is in constrast to active fiber cavities that may contain a gain medium such as an erbium doped fiber amplifier and can be used to create a fiber ring laser.

The field in a fiber cavity will experience losses, both due to propagation (intrinsic absorption) and output coupling. The condition when the absorption losses are equal to the coupling losses is known as critical coupling and allows for the complete extraction of the output field when the pump frequency is resonant. A low power sweep of the pump frequency across the resonance will then result in the pump field power showing a dip with zero transmission on resonance. The frequency separation between the pump laser and the resonant frequency of the pump mode is referred to as the pump detuning.

The intracavity field will interfere constructively with itself and be resonant with the cavity whenever the length $L$ of the resonator circumference corresponds to an integer number $m$ of wavelengths. This is expressed by the condition $\beta_{m} L=2 \pi m$, where 
$\beta_{m}=k_{m} n_{\text {eff }}$ is the propagation constant of the mode with vacuum wavenumber $k_{m}=$ $2 \pi / \lambda_{m}$ and effective refractive index $n_{\text {eff }}$. The resonances are in the absence of dispersion separated by an equidistant frequency spacing known as the free-spectral-range (FSR) given by $\Delta v_{F S R}=1 /\left(\beta_{1} L\right)$, with $\beta_{1}=d \beta_{m} /\left.d \omega\right|_{\omega_{m}}=(1 / c)(n+\omega d n / d \omega)$ being the inverse group velocity.

Resonators are commonly characterized either by their quality factor or their optical finesse. The finesse $\mathcal{F}$ is inversely proportional to the losses as $\mathcal{F}=\pi / \alpha$ and is a measure of the ratio between the FSR and the resonance linewidth $\Delta v$, i.e., $\mathcal{F}=\Delta v_{F S R} / \Delta v$. Meanwhile, the quality or Q-factor measures the ratio between the frequency and the linewidth, i.e., $Q=v / \Delta \nu$, and is related to the finesse as $\mathcal{F}=Q \lambda \Delta v_{F S R} / c$. The $Q$-factor is further related to the photon lifetime which is the characteristic decay time of the mode by $Q=\omega t_{p h}$.

We now consider the temporal evolution of the intracavity field in the so-called meanfield approximation. This approximation assumes that the field envelope changes little between each round trip so that the input pump field and the coupling losses can be taken as being distributed along the length of the cavity. The mean-field approximation is particularly convenient since it allows for the evolution of the field over multiple round trips to be modeled by a single partial differential equation in the form of a driven and damped nonlinear Schrödinger (NLS) equation. The validity of this approximation and the derivation of the evolution equation will be considered further in the next section.

The driven and damped NLS equation for the slowly varying envelope $E$ of the intracavity electric field in a dispersive fiber ring cavity is given by [80]

$$
t_{R} \frac{\partial E(t, \tau)}{\partial t}+i \frac{\beta_{2} L}{2} \frac{\partial^{2} E(t, \tau)}{\partial \tau^{2}}-i \gamma L|E(t, \tau)|^{2} E(t, \tau)=-\left(\alpha+i \delta_{0}\right) E(t, \tau)+\sqrt{\theta} E_{\text {in }}
$$

where $t_{R}=1 / \Delta v_{F S R}$ is the round trip time, $\alpha$ the total round trip loss, $\delta_{0}$ the pump detuning, $\theta$ the (intensity) coupling coefficient and $E_{i n}$ the driving field. Equation (1.32) is written using two different time-scales, with a fast time $\tau$ corresponding to the ordinary retarded time for a pulse moving at the group velocity and a separate slow time $t$ that measures the evolution of the field over multiple round trips. Equation (1.32) is also known as the Lugiato-Lefever equation (LLE) since it is formally equivalent to a model originally used for describing spatially transverse structures in diffractive and dissipative nonlinear cavities [81]. The LLE has also recently been used for modeling the formation of optical frequency combs in crystalline whispering-gallery-mode resonators and glass-based microring resonators [82,83]. These are very similar to fiber ring resonators in most regards except for their smaller dimensions and larger FSR. Note that the LLE is often generalized by including higher-order dispersion and other effects such as self-steepening and Raman scattering when describing broadband fields.

The constant CW solution of Eq. (1.32) is found by setting the derivative terms to zero and is given by $E_{0}=\sqrt{\theta} E_{i n} /\left(\alpha+i\left(\delta_{0}-\gamma L\left|E_{0}\right|^{2}\right)\right)$, where the intracavity power $\left|E_{0}\right|^{2}$ satisfies the bistable cubic equation

$$
\theta\left|E_{i n}\right|^{2}=\left|E_{0}\right|^{2}\left[\left(\delta_{0}-\gamma L\left|E_{0}\right|^{2}\right)^{2}+\alpha^{2}\right]
$$

This equation has either one or three simultaneous real solutions depending on the pump detuning. It is single valued for $\delta_{0} \leq \sqrt{3} \alpha$ and displays bistability with three solutions for $\delta_{0}>\sqrt{3} \alpha$, see Figure 1.12. The middle branch of the response 


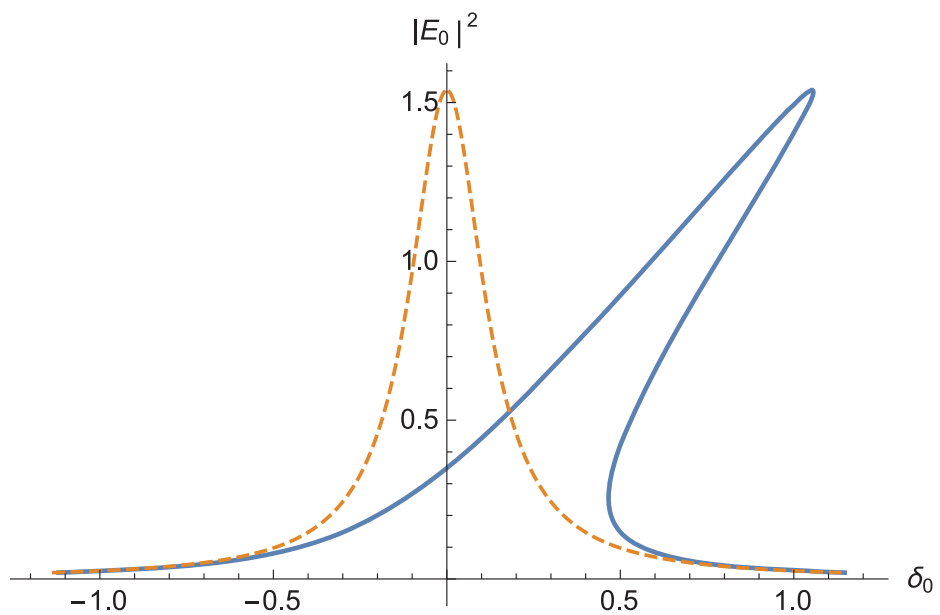

Figure 1.12 Bistability of the continuous wave solution induced by nonlinear Kerr tilt. The solid line shows the resonance bistability for the intracavity power as a function of the detuning parameter while the dashed line is the corresponding resonance in the absence of any nonlinearity $(\gamma \rightarrow 0)$. Parameters $\alpha=\theta=0.13, \gamma=1.8 \mathrm{~W}^{-1} \mathrm{~km}^{-1}, L=380 \mathrm{~m}$ and $P_{\text {in }}=200 \mathrm{~mW}$.

curve is, however, always unstable to $\mathrm{CW}$ perturbations and is not observable in practice.

In the presence of dispersion, the driven and damped NLS Eq. (1.32) can also exhibit modulational instability with qualitatively new features compared to propagation in a straight length of fiber due to the extra degree of freedom provided by the detuning. In particular, one finds that Eq. (1.32) can display modulational instability not only for anomalous dispersion, but also in the normal dispersion regime $[80,84]$. To analyse the stability we look for a perturbed solution of the form $E=\left(\left|E_{0}\right|+u(t, \tau)+\right.$ $i v(t, \tau)) e^{i \operatorname{Arg}\left\{E_{0}\right\}}$ and linearize Eq. (1.32) around the steady-state CW solution to obtain

$$
t_{R}\left(\tilde{u}_{t}+i \tilde{v}_{t}\right)-i \frac{\beta_{2} L}{2} \omega^{2}(\tilde{u}+i \tilde{v})-i \gamma L\left|E_{0}\right|^{2}(3 \tilde{u}+i \tilde{v})=-\left(\alpha+i \delta_{0}\right)(\tilde{u}+i \tilde{v})
$$

where again the tilde denotes the Fourier transform with respect to $\tau$. The real and imaginary part of this expression provides two separate linear equations and the potential growth of the perturbations can be investigated by studying the eigenvalues of their coefficient matrix. Performing the calculation, one finds that the eigenvalues are given by the expression

$$
\lambda=-\alpha \pm \sqrt{\left(\gamma L\left|E_{0}\right|^{2}\right)^{2}-\left(\delta_{0}-\left(\beta_{2} L / 2\right) \omega^{2}-2 \gamma L\left|E_{0}\right|^{2}\right)^{2}} .
$$

Modulational instability is observed whenever the real part of an eigenvalue is positive. The maximum growth rate is $\lambda_{\max }=\gamma L\left|E_{0}\right|^{2}-\alpha$ and is found for frequencies satisfying the condition that the wavevector mismatch $\Delta k=\delta_{0}-\left(\beta_{2} L / 2\right) \omega^{2}-2 \gamma L\left|E_{0}\right|^{2}$ is equal to zero, i.e., $\omega_{\text {max }}^{2}=\left(2 / \beta_{2} L\right)\left(\delta_{0}-2 \gamma L\left|E_{0}\right|^{2}\right)$. Contrary to the case of propagation in a straight fiber we see that this equation can have real solutions, and thus be modulational unstable, also in the normal dispersion regime when $\beta_{2}>0$, provided that the detuning $\delta_{0}>2 \gamma L\left|E_{0}\right|^{2}$. From the maximum growth rate we also find that there is a minimum 
power threshold for the instability to occur, namely, $\left|E_{0}\right|^{2} \geq \alpha /(\gamma L)$. This threshold can be understood to signify that the parametric gain must balance the round trip losses.

To further investigate the influence of the detuning we introduce $g_{ \pm}=2 \gamma L\left|E_{0}\right|^{2} \pm$ $\sqrt{\left(\gamma L\left|E_{0}\right|^{2}\right)^{2}-\alpha^{2}}$ and write the threshold condition $\lambda=0$ for the instability as $\left(\beta_{2} L / 2\right) \omega^{2}=\delta_{0}-g_{ \pm}$. For anomalous dispersion $\left(\beta_{2}<0\right)$ we must then require a detuning $\delta_{0}<g_{+}$for MI to occur, while we should have $\delta_{0}>g_{-}$for normal dispersion $\left(\beta_{2}>0\right)$.

It should, however, be kept in mind that the stability analysis involves the power of the intracavity field, which is related to the pump field though Eq. (1.33), rather than the experimentally accessible power of the pump field itself. This means that even though MI can occur also in the normal dispersion regime, it will not arise spontaneously unless the detuning and power are in the correct range. Beyond the initial growth of the sidebands the subsequent dynamics of the modulational instability within the dissipative cavity can in different regimes give rise not only to unstable MI, but also to the formation of stable patterns of periodic temporal structures as well as localized cavity soliton solutions, $\mathrm{cf}$. Section 1.4.3.

\subsubsection{Parametric Resonances and Period Doubling Phenomena}

The mean-field equation of the previous section is very convenient for modeling the cavity dynamics and the formation of frequency combs when the intracavity field is changing slowly. More generally the evolution of the field inside a fiber ring resonator can be modeled using an infinite-dimensional Ikeda map [85] that consists of an ordinary NLS equation for the propagation of the field inside the fiber waveguide, together with boundary conditions that relates the fields between each round trip. The evolution of the slowly varying field envelope at round trip $m$ is then described by $[80,86,87]$

$$
\begin{aligned}
& E^{m+1}(\tau, 0)=\sqrt{\theta} E_{i n}+\sqrt{1-\theta} e^{i \phi_{0}} E^{m}(\tau, L) \\
& \frac{\partial E^{m}(\tau, z)}{\partial z}=-\frac{\alpha_{i}}{2} E^{m}(\tau, z)-i \frac{\beta_{2}}{2} \frac{\partial^{2} E^{m}(\tau, z)}{\partial \tau^{2}}+i \gamma\left|E^{m}(\tau, z)\right|^{2} E^{m}(\tau, z)
\end{aligned}
$$

where $z$ is the coordinate along the circumference of the fiber, $\alpha_{i}$ is the intrinsic fiber loss $\left(\alpha=\left(\alpha_{i} L+\theta\right) / 2\right)$ and $\phi_{0}=2 \pi l-\delta_{0}$ is the linear phase-shift of the pump mode, which is assumed to correspond to longitudinal mode number $l=0$. The driven and damped NLS Eq. (1.32) is obtained by averaging this map over one round trip, which is permissible if the detuning is small $\delta_{0} \ll 1$ and the characteristic nonlinear length scale $L_{n l}=1 /\left(\gamma|E|^{2}\right)$ is much longer than the cavity length $L$. However, if the intracavity field changes appreciably over a single round trip, it is necessary to consider the dynamics using the full map Eqs. $(1.36,1.37)$. As we shall see, the presence of the boundary conditions will in fact give rise to new instabilities which leads to phenomena such as period doubling that cannot be modeled using the simple mean-field theory $[86,88]$.

The CW solution of the map that is periodically restored after each round trip is a fixed point of the equation $E_{0}=\rho e^{\phi} E_{0}+\sqrt{\theta} E_{\text {in }}$ with $\rho=\sqrt{1-\theta} e^{-\alpha_{i} L / 2}, \phi=\delta_{0}-\gamma L_{\text {eff }}\left|E_{0}\right|^{2}$ and $L_{e f f}=\left(1-e^{-\alpha_{i} L}\right) / \alpha_{i}$. The intracavity power satisfies an equation similar to Eq. (1.33), namely

$$
\theta\left|E_{i n}\right|^{2}=\left|E_{0}\right|^{2}\left[4 \rho \sin ^{2}(\phi / 2)+(1-\rho)^{2}\right] .
$$




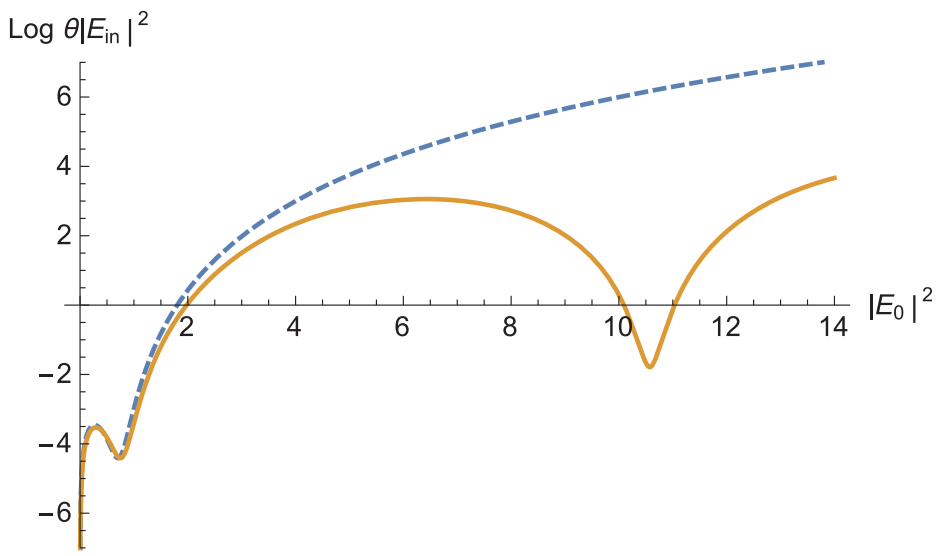

Figure 1.13 Comparison of pump dependence on the intracavity power for multi-valued stationary continuous wave solutions of the LLE (dashed) and the lkeda map (solid). Parameters $\alpha=\theta=0.13$, $\gamma=1.8 \mathrm{~W}^{-1} \mathrm{~km}^{-1}, L=380 \mathrm{~m}$ and $\delta_{0}=0.5$.

However, contrary to the mean-field case, this equation is not only bistable, but multistable and may have additional simultaneous real solutions, cf. Figure 1.13. In fact, it is easily seen that resonances occur whenever the nonlinear phase $\phi\left(\left|E_{0}\right|^{2}\right)$ is an integer multiple of $2 \pi$.

The stability of the CW solution of the map Eqs. $(1.36,1.37)$ can be analysed by linearizing Eq. (1.37) while assuming a perturbation of the form $E^{m}(\tau, z)=\left[E_{0}+u^{m}(\tau, z)+\right.$ $\left.i v^{m}(\tau, z)\right] \exp \left[-\alpha_{i} z / 2+i \gamma\left(1-e^{-\alpha_{i} z}\right)\left|E_{0}\right|^{2} / \alpha_{i}+i \operatorname{Arg}\left\{E_{0}\right\}\right]$. The Fourier transform of the real and imaginary part of the perturbation functions $w^{m}(z)=\left[\tilde{u}^{m}(\omega, z), \tilde{v}^{m}(\omega, z)\right]^{T}$ is then found to satisfy the linear equation system

$$
\frac{d w^{m}}{d z}=\left[\begin{array}{cc}
0 & -\left(\beta_{2} / 2\right) \omega^{2} \\
\left(\beta_{2} / 2\right) \omega^{2}+2 \gamma\left|E_{0}\right|^{2} e^{-\alpha_{i} z} & 0
\end{array}\right] w^{m} .
$$

In the absence of absorption losses, i.e., $\alpha_{i} \rightarrow 0$, the coefficient matrix becomes independent of $z$ which allows the eigenvalues to be calculated explicitly in order to recover the familiar result for the modulational instability gain in a lossless fiber described by the NLS equation (cf. Eq. (1.3)), namely

$$
\mu=\omega \sqrt{-\beta_{2} \gamma\left|E_{0}\right|^{2}-\left(\beta_{2} / 2\right)^{2} \omega^{2}} .
$$

Although it is possible to analytically calculate the instability gain of the Ikeda map for the case when the absorption losses are ignored, it is generally simpler to use numerical Floquet analysis to investigate the stability [87]. The Floquet analysis is based on investigating the eigenvalues of the fundamental matrix $W=\left[w_{1}^{m+1}(0), w_{2}^{m+1}(0)\right]$. This fundamental matrix can be obtained by first integrating Eq. (1.39) numerically over one round trip for two independent initial conditions, e.g., $w_{1,2}^{m}(0)=[1,0]^{T},[0,1]^{T}$, in order to find $w_{1,2}^{m}(L)$ before applying the boundary condition Eq. (1.36) to finally get $w_{1,2}^{m+1}(0)$.

Contrary to the case of the driven and damped NLS Eq. (1.32), the Ikeda map Eqs. $(1.36,1.37)$ has multiple instability bands for high intracavity power that form so-called resonance tongues, see Figure 1.14 in the color plate section. These occur alternatively under both resonant and anti-resonant conditions. For the resonant case 


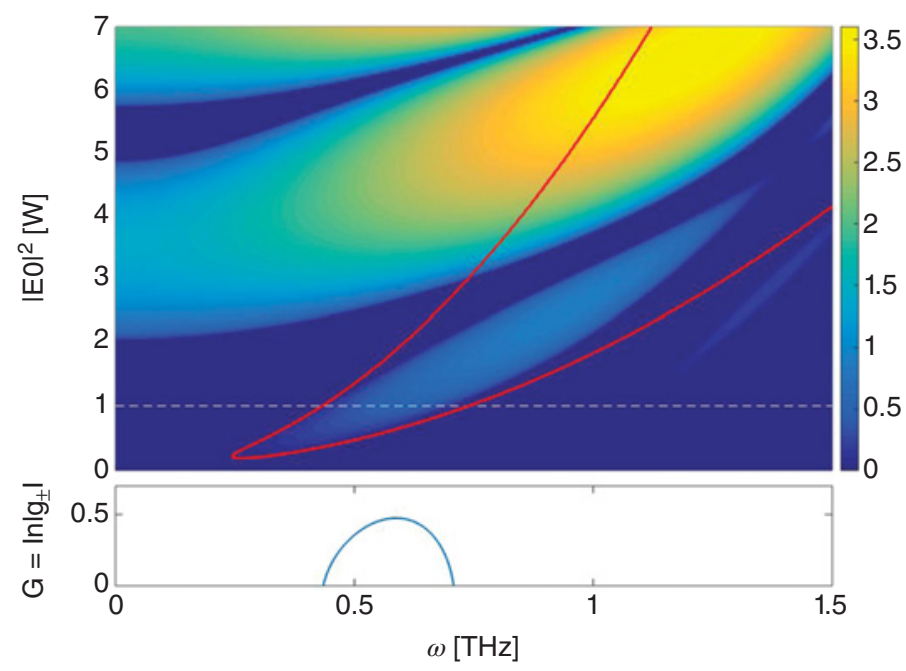

Figure 1.14 Parametric instability tongues of the Ikeda map for anomalous dispersion. The red contour shows the predicted range of modulational instability for the LLE. Below a cross-section corresponding to the dashed line is shown with the Ml growth rate. Parameters $\alpha=\theta=0.13, \beta_{2}=-20 \mathrm{ps}^{2} \mathrm{~km}^{-1}$,

$\gamma=1.8 \mathrm{~W}^{-1} \mathrm{~km}^{-1}, L=380 \mathrm{~m}$ and $\delta_{0}=0$. For a color version of this figure please see color plate section.

the unstable perturbation repeats itself periodically each round trip, while for the antiresonant case the perturbation will be $\pi$ out of phase after one round trip and will not recover its original phase until two round trips have elapsed. The latter case is a form of period doubling instability which is referred to as P2-MI to distinguish it from the ordinary resonant instability or CW-MI [86]. The P2-MI is in fact usually the first instability to occur, i.e., the instability with the lowest power threshold, for normal dispersion fibers where the detuning has not been exploited in order to achieve phase matching for the lowest order CW-MI tongue that corresponds to the mean-field MI considered in the previous section.

Finally, we point out that parametric instabilities of similar origin might occur also in the driven and damped NLS Eq. (1.32) in the presence of dispersion (or nonlinearity) intracavity management. In this case the periodicity can occur on a scale $1 / n, n=1,2, \ldots$ in units of the cavity length, and for odd $n$ the instability is of the P2-MI type [89]. Recently, such a cavity has been implemented and employed to observe, for the first time, the steady-state excitation of sidebands on the lower branch due to the meanfield MI (temporal Turing instability) in the normal dispersion regime. This instability competes with the parametric (Faraday) instability on the upper branch of the bistable response induced by the dispersion map [90].

\subsubsection{FWM in a Fiber Cavity for Optical Buffer Applications}

In this section we briefly consider an application of the four-wave mixing process in passive fiber cavities. This application relies on the fact that the growth rate of the cavity MI is purely real and can give rise to stationary periodic patterns and temporal cavity solitons. The cavity solitons are a special kind of localized pulse structures that can be found in driven and damped nonlinear cavities [91]. The spectral signature of a temporal cavity soliton is a mode-locked optical frequency comb having the frequency spacing 

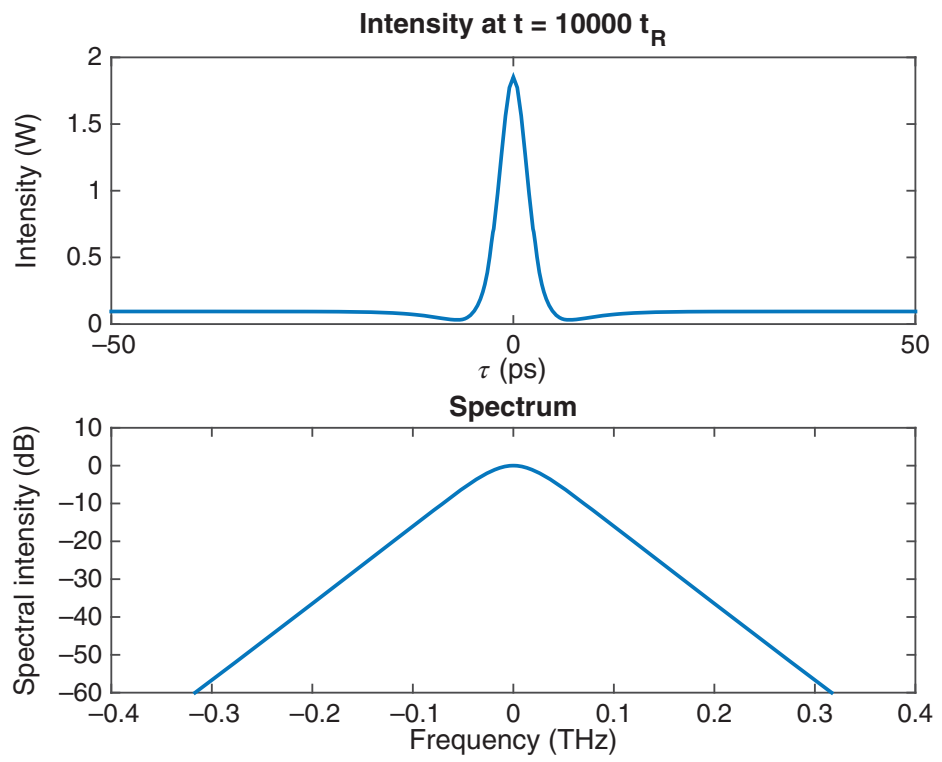

Figure 1.15 Intensity profile and normalized spectrum for a stationary temporal cavity soliton obtained from numerical solution of Eq. (1.32). The dense mode spectrum is treated as a continuum and only a portion of the round trip duration is simulated in order to simultaneously achieve sufficient temporal and spectral resolution with only 4096 sampling points. Note that the soliton is much less energetic than the continuous wave background so the latter has been filtered out from the spectrum. Parameters $\alpha=\theta=0.13, \beta_{2}=-20 \mathrm{ps}^{2} \mathrm{~km}^{-1}, \gamma=1.8 \mathrm{~W}^{-1} \mathrm{~km}^{-1}, L=380 \mathrm{~m}, \delta_{0}=0.5$ and $P_{\text {in }}=150 \mathrm{~mW}$.

of a single FSR. Like other temporal solitons they represent a balance between dispersion and nonlinearity, but being dissipative structures they also simultaneously balance the gain originating from the driving beam with losses due to absorption and output coupling. An example of a cavity soliton solution of Eq. (1.32) is shown in Figure 1.15. The cavity soliton coexists with a constant low-intensity $\mathrm{CW}$ background corresponding to the lowest lying solution branch of Eq. (1.33) that is stable against MI, and can be considered as a compound object of a soliton plus background that is a form of lowdimensional dynamical attractor.

For a given set of pump parameters, i.e., pump power and detuning, there is a single type of cavity soliton with both amplitude and width proportional to the square root of the detuning. The solitons are phase-locked to the pump in such a way that the nonlinear phase-shift compensates for the pump field detuning. Although not integrable, these solitons are remarkably robust against different sorts of perturbations which suggests that they may be suitable candidates for use as optical bits to encode binary data [92]. By dividing the round trip time of the cavity into time slots and using cavity solitons to indicate logical one and the absence of a soliton, the logical zero, it is possible to form an optical buffer memory that in the presence of the pump beam can hold an arbitrary bit pattern for a potentially indefinite amount of time. This has possible applications, e.g., for all-optical signal processing which can benefit from the higher speeds afforded by optics in comparison to electronics. 
A benefit of an all-optical bit buffer using cavity solitons in a passive fiber cavity is that it will, contrary to an active system where amplification is used, not suffer from signal degradation due to the accumulation of noise. Moreover, the soliton formation process is rather robust, which allows for pulse reshaping and wavelength conversion. The excitation of individual cavity solitons can be accomplished using an addressing beam with pulses that are close in shape and frequency to the final soliton profile. Numerical simulations have shown that such pulses can simultaneously both write and erase cavity solitons in a way that is reminiscent of XOR (eXclusive OR) gate logic.

The first experimental demonstration of temporal Kerr cavity solitons was performed by Leo et al. in 2010 [93]. In their experiment they used a passive fiber ring resonator made of $380 \mathrm{~m}$ of standard single-mode telecom fiber and having a round trip time of $1.85 \mu$ s to demonstrate the stable storage of individual cavity solitons and cavity soliton patterns without distortion for a time duration of over $1 \mathrm{~s}$. The system was pumped with $225 \mathrm{~mW}$ of power using an ultra-narrow linewidth continuous wave laser operating at $1550 \mathrm{~nm}$ and the 4 ps cavity soliton pulses were characterized using both direct time domain oscilloscope measurements as well as frequency domain measurements using an optical spectrum analyzer. To excite the cavity solitons an additional mode-locked fiber laser was used to produce writing pulses that could address individual time slots. The writing process was confirmed to be very robust, as well as wavelength- and phaseinsensitive, so that the addressing pulse did not have to precisely match the cavity soliton profile. The particular configuration studied was estimated to allow for the potential alloptical storage of up to 45000 bits at a bitrate of $25 \mathrm{Gbit} / \mathrm{s}$.

The maximum storage time of the cavity solitons in the above experiment was limited by environmental perturbations. However, an additional limit is also set by the interactions due to the tail overlap between individual solitons that can lead to either attractive or repulsive behavior when the solitons are closely spaced in time. This relative motion of soliton pairs can result in timing-jitter and even the annihilation of solitons due to collisions. Various method for controlling these interactions have been proposed: it has, for example, been shown that they can be completely suppressed by the injection of CW light above a certain amplitude [94]. Alternatively, by introducing a shallow periodic modulation, it has been found that the solitons will be attracted to the peaks of the modulation phase profile [95]. Yet another method of suppressing the interactions between cavity solitons is using bichromatic pumping. Two pumps with equal amplitude and phase but with a frequency separation corresponding to a multiple of the FSR have also been shown to be capable of supporting cavity solitons [96]. In this case there is no continuous wave background and the solitons are superimposed on a periodic low intensity pattern with the motion of the solitons constrained by the need for them to remain on the crests of the pattern.

\section{References}

1 Bespalov, V. and Talanov, V. (1966) Filamentary structure of light beams in nonlinear liquids. ZhETF Pisma Redaktsiiu, 3, 471 (JETP Lett. 3 (1966) 307).

2 Benjamin, T.B. and Feir, J. (1967) The disintegration of wave trains on deep water part 1. theory. Journal of Fluid Mechanics, 27, 417-430, DOI:10.1017/S002211206700045X. 
3 Dudley, J.M., Genty, G., and Coen, S. (2006) Supercontinuum generation in photonic crystal fiber. Reviews of Modern Physics, 78, 1135-1184, DOI:10.1103/RevModPhys.78. 1135.

4 Dudley, J.M., Genty, G., Dias, F., Kibler, B., and Akhmediev, N. (2009) Modulation instability, Akhmediev Breathers and continuous wave supercontinuum generation. Optics Express, 17, 21 497-21 508, DOI:10.1364/OE.17.021497.

5 Solli, D., Ropers, C., Koonath, P., and Jalali, B. (2007) Optical rogue waves. Nature, 450, 1054-1057, DOI:10.1038/nature06402.

6 Tai, K., Tomita, A., Jewell, J.L., and Hasegawa, A. (1986) Generation of subpicosecond solitonlike optical pulses at $0.3 \mathrm{THz}$ repetition rate by induced modulational instability. Applied Physics Letters, 49, 236-238, DOI:10.1063/1.97181.

7 Fermi, E., Pasta, J., and Ulam, S. (1955) Studies of nonlinear problems, Los Alamos Scientific Laboratory Report No. LA-1940, Los Alamos, New Mexico.

8 Zabusky, N.J. and Kruskal, M.D. (1965) Interaction of "solitons" in a collisionless plasma and the recurrence of initial states. Physical Review Letters, 15, 240-243, DOI:10.1103/ PhysRevLett.15.240.

9 Akhmediev, N. and Korneev, V. (1986) Modulation instability and periodic solutions of the nonlinear Schrödinger equation. Theoretical and Mathematical Physics, 69, 1089-1093, DOI:10.1007/BF01037866.

10 Akhmediev, N., Korneev, V., and Mitskevich, N. (1988) N-modulation signals in a single-mode optical waveguide under nonlinear conditions. Soviet Physics JETP, 67, 89.

11 Akhmediev, N., Eleonskii, V., and Kulagin, N. (1987) Exact first-order solutions of the nonlinear Schrödinger equation. Theoretical and Mathematical Physics, 72, 809-818, DOI:10.1007/BF01017105.

12 Ablowitz, M.J. and Herbst, B. (1990) On homoclinic structure and numerically induced chaos for the nonlinear Schrödinger equation. SIAM Journal of Applied Mathematics, 50, 339-351, DOI:10.1137/0150021.

13 Lake, B.M., Yuen, H.C., Rungaldier, H., and Ferguson, W.E. (1977) Nonlinear deep-water waves: theory and experiment. part 2. evolution of a continuous wave train. Journal of Fluid Mechanics, 83, 49-74, DOI:10.1017/S0022112077001037.

14 Chabchoub, A., Hoffmann, N.P., and Akhmediev, N. (2011) Rogue wave observation in a water wave tank. Physical Review Letters, 106, 204 502, DOI:10.1103/PhysRevLett.106. 204502.

15 Van Simaeys, G., Emplit, P., and Haelterman, M. (2001) Experimental demonstration of the Fermi-Pasta-Ulam recurrence in a modulationally unstable optical wave. Physical Review Letters, 87, 033 902, DOI:10.1103/PhysRevLett.87.033902.

16 Van Simaeys, G., Emplit, P., and Haelterman, M. (2002) Experimental study of the reversible behavior of modulational instability in optical fibers. Journal of the Optical Society of America B, 19, 477-486, DOI:10.1364/JOSAB.19.000477.

17 Zhao, B., Tang, D., and Tam, H. (2003) Experimental observation of FPU recurrence in a fiber ring laser. Optics Express, 11, 3304-3309, DOI:10.1364/OE.11.003304.

18 Hammani, K., Wetzel, B., Kibler, B., et al. (2011) Spectral dynamics of modulation instability described using Akhmediev breather theory. Optics Letters, 36, 2140-2142, DOI:10.1364/OL.36.002140.

19 Kibler, B., Fatome, J., Finot, C., et al. (2010) The Peregrine soliton in nonlinear fibre optics. Nature Physics, 6, 790-795, DOI:10.1038/nphys1740. 
20 Kibler, B., Fatome, J., Finot, C., et al. (2012) Observation of Kuznetsov-Ma soliton dynamics in optical fibre. Scientific Reports, 2, 463, DOI:10.1038/srep00463.

21 Beeckman, J., Hutsebaut, X., Haelterman, M., and Neyts, K. (2007) Induced modulation instability and recurrence in nematic liquid crystals. Optics Express, 15, 11 185-11 195, DOI:10.1364/OE.15.011185.

$22 \mathrm{Wu}, \mathrm{M}$. and Patton, C.E. (2007) Experimental observation of Fermi-Pasta-Ulam recurrence in a nonlinear feedback ring system. Physical Review Letters, 98, 047 202, DOI:10.1103/PhysRevLett.98.047202.

23 Farota, A.K. and Faye, M.M. (2013) Experimental study of the Fermi-Pasta-Ulam recurrence in a bi-modal electrical transmission line. Physica Scriptai, 88, 55 802-55 805, DOI:10.1088/0031-8949/88/05/055802.

24 Infeld, E. (1981) Quantitive theory of the Fermi-Pasta-Ulam recurrence in the nonlinear Schrödinger equation. Physical Review Letters, 47, 717-718, DOI:10.1103/PhysRevLett. 47.717.

25 Cappellini, G. and Trillo, S. (1991) Third-order three-wave mixing in single-mode fibers: exact solutions and spatial instability effects. Journal of the Optical Society of America B, 8, 824-838, DOI:10.1364/JOSAB.8.000824.

26 Trillo, S. and Wabnitz, S. (1991) Dynamics of the nonlinear modulational instability in optical fibers. Optics Letters, 16, 986-988, DOI:10.1364/OL.16.000986.

27 Trillo, S. and Wabnitz, S. (1991) Self-injected spatial mode-locking and coherent all-optical AM/FM switching based on modulational instability. Optics Letters, 16, 1566-1568, DOI:10.1364/OL.16.001566.

28 Wabnitz, S. (1988) Modulational polarization instability of light in a nonlinear birefringent dispersive medium. Physical Review, A, 38, 2018-2021, DOI:10.1103/ PhysRevA.38.2018.

29 Trillo, S. and Wabnitz, S. (1991) Nonlinear modulation of coupled waves in birefringent optical fibers. Physics Letters, A, 159, 252-256, DOI:10.1016/0375-9601(91)90519-E.

30 Cappellini, G. and Trillo, S. (1991) Energy conversion in degenerate four-photon mixing in birefringent fibers. Optics Letters, 16 (12), 895-897, DOI:10.1364/OL.16.000895. URL:http://ol.osa.org/abstract.cfm?URI=ol-16-12-895.

31 Cappellini, G. and Trillo, S. (1991) Bifurcations and three-wave-mixing instabilities in nonlinear propagation in birefringent dispersive media. Physical Review, A, 44, 7509-7523, DOI:10.1103/PhysRevA.44.7509. URL:http://link.aps.org/doi/10.1103/ PhysRevA.44.7509.

32 Drummond, P., Kennedy, T., Dudley, J., Leonhardt, R., and Harvey, J. (1990) Cross-phase modulational instability in high-birefringence fibers. Optics Communications, 78 (2), 137-142. URL:http://dx.doi.org/10.1016/0030-4018(90)90110-F.

33 Rothenberg, J.E. (1990) Modulational instability for normal dispersion. Physical Review, A, 42, 682-685, DOI:10.1103/PhysRevA.42.682. URL:http://link.aps.org/doi/10.1103/ PhysRevA.42.682.

34 De Angelis, C., Trillo, S., and Santagiustina, M. (1994) Induced modulational instability in high-birefringence fibers: the strong conversion regime. Optics Letters, 19 (5), 335-337, DOI:10.1364/OL.19.000335. URL:http://ol.osa.org/abstract.cfm?URI=ol-195-335.

35 De Angelis, C., Santagiustina, M., and Trillo, S. (1995) Four-photon homoclinic instabilities in nonlinear highly birefringent media. Physical Review, A, 51, 774-791, DOI:10.1103/PhysRevA.51.774. URL:http://link.aps.org/doi/10.1103/PhysRevA.51.774. 
36 Seve, E., Millot, G., and Trillo, S. (2000) Strong four-photon conversion regime of cross-phase-modulation-induced modulational instability. Physical Review, E, 61, 3139-3150, DOI:10.1103/PhysRevE.61.3139. URL:http://link.aps.org/doi/10.1103/ PhysRevE.61.3139.

37 Agrawal, G.P. (1987) Modulation instability induced by cross-phase modulation. Physical Review Letters, 59, 880-883, DOI:10.1103/PhysRevLett.59.880.

URL:http://link.aps.org/doi/10.1103/PhysRevLett.59.880.

38 Thompson, J.R. and Roy, R. (1991) Nonlinear dynamics of multiple four-wave mixing processes in a single-mode fiber. Physical Review, A, 43, 4987-4996, DOI:10.1103/ PhysRevA.43.4987. URL:http://link.aps.org/doi/10.1103/PhysRevA.43.4987.

39 Rothenberg, J.E. (1990) Modulational instability of copropagating frequencies for normal dispersion. Physical Review Letters, 64, 813-813, DOI:10.1103/PhysRevLett.64. 813. URL:http://link.aps.org/doi/10.1103/PhysRevLett.64.813.

40 Armaroli, A. and Trillo, S. (2014) Modulational instability due to cross-phase modulation versus multiple four-wave mixing: the normal dispersion regime. Journal of the Optical Society of America B, 31, 551-558, DOI:10.1364/JOSAB.31.000551.

41 Trillo, S., Wabnitz, S., and Kennedy, T.A.B. (1994) Nonlinear dynamics of dual-frequency-pumped multiwave mixing in optical fibers. Physical Review, A, 50 (2), 1732-1747, DOI:10.1103/PhysRevA.50.1732. URL:http://link.aps.org/doi/10.1103/ PhysRevA.50.1732.

42 Armaroli, A. and Trillo, S. (2011) Collective modulation instability of multiple four-wave mixing. Optics Letters, 36, 1999-2001, DOI:10.1364/OL.36.001999.

43 Fatome, J., Finot, C., Armaroli, A., and Trillo, S. (2013) Observation of modulationally unstable multi-wave mixing. Optics Letters, 38, 181-183, DOI:10.1364/OL.38.000181.

44 Dudley, J.M., Dias, F., Erkintalo, M., and Genty, G. (2014) Instabilities, breathers and rogue waves in optics. Nature Photonics, 8, 755-764, DOI:10.1038/nphoton.2014.220.

45 Erkintalo, M., Genty, G., Wetzel, B., and Dudley, J.M. (2011) Akhmediev breather evolution in optical fiber for realistic initial conditions. Physics Letters, A, 375 (19), 2029-2034. URL:http://dx.doi.org/10.1016/j.physleta.2011.04.002.

46 Millot, G., Seve, E., Wabnitz, S., and Trillo, S. (1998) Observation of a novel large-signal four-photon instability in optical wave mixing. Physical Review Letters, 80, 504-507, DOI:10.1103/PhysRevLett.80.504. URL:http://link.aps.org/doi/10.1103/PhysRevLett. 80.504.

47 Bendahmane, A., Mussot, A., Kudlinski, A., et al. (2015) Optimal frequency conversion in the nonlinear stage of modulation instability. Optics Express, 23 (24), 30 861-30 871, DOI:10.1364/OE.23.030861. URL:http://www.opticsexpress.org/abstract.cfm?URI=oe23-24-30861.

48 Wabnitz, S. and Akhmediev, N. (2010) Efficient modulation frequency doubling by induced modulation instability. Optics Communications, 283, 1152-1154, DOI:10.1016/j.optcom.2009.11.030.

49 Akhmediev, N., Eleonskii, V., and Kulagin, N. (1985) Generation of periodic trains of picosecond pulses in an optical fiber: exact solutions. Soviet Physics JETP, 62, 894-899.

50 Erkintalo, M., Hammani, K., Kibler, B., et al. (2011) Higher-order modulation instability in nonlinear fiber optics. Physical Review Letters, 107, 253 901, DOI:10.1103/ PhysRevLett.107.253901.

51 Fatome, J., Mansouri, I.E., Blanchet, J.L., et al. (2013) Even harmonic pulse train generation by cross-polarization-modulation seeded instability in optical fibers. Journal of the Optical Society of America B, 30, 99-106, DOI:10.1364/JOSAB.30.000099. 
52 Soto-Crespo, J.M., Ankiewicz, A., Devine, N., and Akhmediev, N. (2012) Modulation instability, Cherenkov radiation, and Fermi-Pasta-Ulam recurrence. Journal of the Optical Society of America B, 29, 1930-1936, DOI:10.1364/JOSAB.29.001930.

53 Mussot, A., Kudlinski, A., Droques, M., Szriftgiser, P., and Akhmediev, N. (2014) Fermi-Pasta-Ulam recurrence in nonlinear fiber optics: the role of reversible and irreversible losses. Physical Review, X, 4, 011 054, DOI:10.1103/PhysRevX.4.011054.

54 Solli, D., Herink, G., Jalali, B., and Ropers, C. (2012) Fluctuations and correlations in modulation instability. Nature Photonics, 6, 463-468, DOI:10.1038/nphoton.2012.126.

55 Wetzel, B., Stefani, A., Larger, L., et al. (2012) Real-time full bandwidth measurement of spectral noise in supercontinuum generation. Scientific Reports, 2, 882, DOI:10.1038/ srep00882.

56 Solli, D.R., Ropers, C., and Jalali, B. (2008) Active control of rogue waves for stimulated supercontinuum generation. Physical Review Letters, 101, 233 902, DOI:10.1103/ PhysRevLett.101.233902.

57 Dudley, J.M., Genty, G., and Eggleton, B.J. (2008) Harnessing and control of optical rogue waves in supercontinuum generation. Optics Express, 16, 3644-3651, DOI:10.1364/OE.16.003644.

58 Nguyen, D.M., Godin, T., Toenger, S., et al. (2013) Incoherent resonant seeding of modulation instability in optical fiber. Optics Letters, 38, 5338-5341, DOI:10.1364/ OL.38.005338.

59 Wabnitz, S. and Wetzel, B. (2014) Instability and noise-induced thermalization of Fermi-Pasta-Ulam recurrence in the nonlinear Schrödinger equation. Physics Letters, $A$, 378, 2750-2756, DOI:10.1016/j.physleta.2014.07.018.

60 Trillo, S. and Wabnitz, S. (1997) Bloch wave theory of modulational polarization instabilities in birefringent optical fibers. Physical Review, E, 56, 1048-1058, DOI:10.1103/PhysRevE.56.1048.

61 Trillo, S. and Wabnitz, S. (1997) Dynamic spontaneous fluorescence in parametric wave coupling. Physical Review, E, 55, R4897-R4900, DOI:10.1103/PhysRevE.55.R4897.

62 Fuerst, R.A., Baboiu, D.M., Lawrence, B., et al. (1997) Spatial modulational instability and multisolitonlike generation in a quadratically nonlinear optical medium. Physical Review Letters, 78, 2756-2759, DOI:10.1103/PhysRevLett.78.2756.

63 Golovchenko, E.A. and Pilipetskii, A.N. (1994) Unified analysis of four-photon mixing, modulational instability, and stimulated Raman scattering under various polarization conditions in fiber. Journal of the Optical Society of America B, 11 (1), 92-101, DOI:10.1364/JOSAB.11.000092. URL:http://josab.osa.org/abstract.cfm?URI=josab11-1-92.

64 McKinstrie, C. and Radic, S. (2004) Phase-sensitive amplification in a fiber. Optics Express, 12 (20), 4973-4979, DOI:10.1364/OPEX.12.004973. URL:http://www.optics express.org/abstract.cfm?URI=oe-12-20-4973.

65 Ettabib, M.A., Jones, L., Kakande, J., et al. (2012) Phase sensitive amplification in a highly nonlinear lead-silicate fiber. Optics Express, 20 (2), 4973-4979, DOI:10.1364/ OE.20.001629. URL:http://www.opticsexpress.org/abstract.cfm?URI=oe-20-2-1629.

66 McKinstrie, C.J., Radic, S., and Chraplyvy, A.R. (2002) Parametric amplifiers driven by two pump waves. IEEE Journal of Selected Topics in Quantum Electronics, 8 (3), 538-547, DOI:10.1109/JSTQE.2002.1016357. URL:http://ieeexplore.ieee.org/stamp/ stamp.jsp?tp=\&arnumber $=1016357$ \&isnumber $=21871$.

67 Marhic, M.E., Yang, F.S., Kazovsky, L.G., and Park, Y. (1996) Widely tunable spectrum translation and wavelength exchange by four-wave mixing in optical fibers. Optics 
Letters, 21 (23), 1906-1908, DOI:10.1364/OL.21.001906. URL:http://ol.osa.org/ abstract.cfm.?URI=ol-21-23-1906.

68 McKinstrie, C., Harvey, J., Radic, S., and Raymer, M. (2005) Translation of quantum states by four-wave mixing in fibers. Optics Express, 13 (22), 9131-9142, DOI:10.1364/ OPEX.13.009131. URL:http://www.opticsexpress.org/abstract.cfm?URI=oe-13-22-9131.

69 McGuinness, H.J., Raymer, M.G., McKinstrie, C.J., and Radic, S. (2010) Quantum frequency translation of single-photon states in a photonic crystal fiber. Physical Review Letters, 105 (9), 093 604, DOI:10.1103/PhysRevLett.105.093604. URL:http://link.aps. org/doi/10.1103/PhysRevLett.105.093604.

70 Krupa, K., Tonello, A., Kozlov, V.V., et al. (2012) Bragg-scattering conversion at telecom wavelengths towards the photon counting regime. Optics Express, 20 (24), 27220 27 225, DOI:10.1364/OE.20.027220. URL:http://www.opticsexpress.org/abstract. cfm?URI=oe-20-24-27220.

71 Provo, R., Murdoch, S., Harvey, J.D., and Méchin, D. (2010) Bragg scattering in a positive $\beta_{4}$ fiber. Optics Letters, 35 (22), 3730-3732, DOI:10.1364/OL.35.003730. URL:http://ol.osa.org/abstract.cfm?URI=ol-35-22-3730.

72 Méchin, D., Provo, R., Harvey, J.D., and McKinstrie, C.J. (2006) 180-nm wavelength conversion based on Bragg scattering in an optical fiber. Optics Express, 14 (20), 8995-8999, DOI:10.1364/OE.14.008995. URL:http://www.opticsexpress.org/abstract. cfm?URI=oe-14-20-8995.

73 Agha, I., Davanço, M., Thurston, B., and Srinivasan, K. (2012) Low-noise chip-based frequency conversion by four-wave-mixing Bragg scattering in $\operatorname{SiN}_{x}$ waveguides. Optics Letters, 37 (14), 2997-2999, DOI:10.1364/OL.37.002997. URL:https://www.osapublish ing.org/ol/abstract.cfm?URI=ol-37-14-2997.

74 Donvalkar, P.S., Venkataraman, V., Clemmen, S., Saha, K., and Gaeta, A.L. (2014) Frequency translation via four-wave mixing Bragg scattering in $\mathrm{Rb}$ filled photonic bandgap fiber. Optics Letters, 39 (6), 1557-1560, DOI:110.1364/OL.39.001557. URL:http://ol.osa.org/abstract.cfm?URI=ol-39-6-1557.

75 McKinstrie, C.J. (2009) Stokes-space formalism for Bragg scattering in a fiber. Optics Communications, 282 (8), 1557-1562, DOI:10.1016/j.optcom.2008.12.066.

URL:http://www.sciencedirect.com/science/article/pii/S0030401808013163.

76 Suchowski, H., Oron, D., Arie, A., and Silberberg, Y. (2008) Geometrical representation of sum frequency generation and adiabatic frequency conversion. Physical Review, A, 78 (6), 063 821, DOI:10.1103/PhysRevA.78.063821. URL:http://link.aps.org/ doi/10.1103/PhysRevA.78.063821.

77 Chen, J., Li, X., and Kumar, P. (2005) Two-photon-state generation via four-wave mixing in optical fibers. Physical Review, A, 72 (3), 033 801, DOI:10.1103/PhysRevA.72.033801. URL:http://link.aps.org/doi/10.1103/PhysRevA.72.033801.

78 Huang, J. and Kumar, P. (1992) Observation of quantum frequency conversion. Physical Review Letters, 68 (14), 2153-2156, DOI:10.1103/PhysRevLett.68.2153.

URL:http://link.aps.org/doi/10.1103/PhysRevLett.68.2153.

79 Collins, M.J., Clark, A.S., He, J., et al. (2012) Low Raman-noise correlated photon-pair generation in a dispersion-engineered chalcogenide As2S3 planar waveguide. Optics Letters, 37 (16), 3393-3395, DOI:10.1364/OL.37.003393. URL:http://ol.osa.org/ abstract.cfm?URI=ol-37-16-3393.

80 Haelterman, M., Trillo, S., and Wabnitz, S. (1992) Dissipative modulation instability in a nonlinear dispersive ring cavity. Optics Communications, 91 (5-6), 401-407. URL:http://dx.doi.org/10.1016/0030-4018(92)90367-Z. 
81 Lugiato, L.A. and Lefever, R. (1987) Spatial dissipative structures in passive optical systems. Physical Review Letters, 58, 2209-2211, DOI:10.1103/PhysRevLett.58.2209.

82 Coen, S., Randle, H.G., Sylvestre, T., and Erkintalo, M. (2013) Modeling of octave-spanning Kerr frequency combs using a generalized mean-field Lugiato-Lefever model. Optics Letters, 38 (1), 37-39. URL:https://doi.org/10.1364/OL.38.000037.

83 Hansson, T., Modotto, D., and Wabnitz, S. (2013) Dynamics of the modulational instability in microresonator frequency combs. Physical Review, A, 88 (2), DOI:10.1103/PhysRevA.88.023819.

84 Haelterman, M., Trillo, S., and Wabnitz, S. (1992) Additive-modulation-instability ring laser in the normal dispersion regime of a fiber. Optics Letters, 17 (10), 745-747. URL:https://doi.org/10.1364/OL.17.000745.

85 Ikeda, K. (1979) Multiple-valued stationary state and its instability of the transmitted light by a ring cavity system. Optics Communications, 30 (2), 257-261, DOI:http://dx. doi.org/10.1016/0030-4018(79)90090-7.

86 Coen, S. and Haelterman, M. (1997) Modulational instability induced by cavity boundary conditions in a normally dispersive optical fiber. Physical Review Letters, 79, 4139-4142, DOI:10.1103/PhysRevLett.79.4139. URL:http://link.aps.org/doi/10.1103/ PhysRevLett.79.4139.

87 Hansson, T. and Wabnitz, S. (2015) Frequency comb generation beyond the Lugiato-Lefever equation: multi-stability and super cavity solitons. Journal of the Optical Society of America B, 32 (7), 1259, DOI:10.1364/JOSAB.32.001259.

88 McLaughlin, D.W., Moloney, J.V., and Newell, A.C. (1985) New class of instabilities in passive optical cavities. Physical review letters, 54 (7), 681. URL:https://doi.org/10.1103/ PhysRevLett.54.681.

89 Conforti, M., Kudlinski, A., Mussot, A., and Trillo, S. (2014) Modulational instability in dispersion oscillating fiber ring cavities. Optics Letters, 39 (14), 4200-4203.

URL:https://doi.org/10.1364/OL.39.004200.

90 Copie, F., Conforti, M., Kudlinski, A., Trillo, S., and Mussot, A. (2016) Competing Turing and Faraday instabilities in longitudinally modulated passive resonators. Physical Review Letters, 116, 143901. URL:https://doi.org/10.1103/PhysRevLett.116.143901.

91 Grelu, P. and Akhmediev, N. (2012) Dissipative solitons for mode-locked lasers. Nature Photonics, 6 (2), 84-92, DOI:10.1038/nphoton.2011.345.

92 McDonald, G.S. and Firth, W.J. (1990) Spatial solitary-wave optical memory. Journal of the Optical Society of America B, 7 (7), 1328-1335. URL:https://doi.org/10.1364/ JOSAB.7.001328.

93 Leo, F., Coen, S., Kockaert, P., Gorza, S.P., Emplit, P., and Haelterman, M. (2010) Temporal cavity solitons in one-dimensional Kerr media as bits in an all-optical buffer. Nature Photonics, 4 (7), 471-476, DOI:10.1038/nphoton.2010.120.

94 Wabnitz, S. (1993) Suppression of interactions in a phase-locked soliton optical memory. Optics Letters, 18 (8), 601-603. URL:https://doi.org/10.1364/OL.18.000601.

95 Luo, K., Jang, J.K., Coen, S., Murdoch, S.G., and Erkintalo, M. (2015) Spontaneous creation and annihilation of temporal cavity solitons in a coherently driven passive fiber resonator. Optics Letters, 40 (16), 3735, DOI:10.1364/OL.40.003735.

96 Hansson, T. and Wabnitz, S. (2014) Bichromatically pumped microresonator frequency combs. Physical Review, A, 90 (1), DOI:10.1103/PhysRevA.90.013811. 